\title{
II. \\ La date de la loi Aebutia
}

\author{
par \\ M. P. F. Girard, \\ professeur à Paris.
}

Il n'y a pas eu, depuis les XII tables, de loi plus décisive pour l'histoire du droit romain que cette loi Acbutia citée seule par Aulu-Gelle et la première par Gaius au sujet de l'abrogation des Actions de la loi et de l'introduction de la procédure formulaire. La question de savoir comment délimiter son domaine en face de celui des lois Juliae a été reprise récenment par $M$. Wlassak et il l'a, je crois, tranchée fort heureusement quant au point essentiel en restreignant le rôle de la loi la plus ancienne à l'admission d'un concours électif entre la procédure nouvelle et la procédure primitive. Mais M. Wlassak qui a entrepris de fixer par des arguments de textes la date relativement moins importante des lois Juliae, s'est abstenu de toute recherche sur celle de la loi Aebutia ${ }^{1}$ ). Je voudrais indiquer ici comment cette loi, ballottée par les commentateurs du commencement du VI ${ }^{\mathbf{e}}$ siècle et même du milieu du Ve à la fin du VII ${ }^{\mathrm{e}}$, peut, à mon sens, se localiser très sûrement, par des raisons tirées du fond du droit, entre des époques extrèmes fort voisines, dans une période étroite

1) Wlassak, Prozessgesetze, 1, 63.86.126. 2, 300. M. Wlassak se borne à constater que la procédure formulaire existe certainement au temps de Cicéron et à admettre comme probable que la loi date de la fiı du VIe siècle ou du commencement du VII e. - Je me contente de relever les principales dates admises par les divers auteurs: an 465 de Rome: Puntschart, Entwicklung d. Civilrechts der Römer, p. 102 et ss., Einfluss d. Gesetzgebung, p. 22 et ss. Ans 513-517: M. Voigt, Ius naturale, 3, 814 et ss., Römische Rechtsgeschichte, 1, 828 et ss. An 520 (Varr. 521): Pighius, suivi par beaucoup d'anciens romanistes. Première moitié du VI e siècle: Burchardi, Wiedereinsetzung, p. 300 et ss. Puchta, Institutionen, $\S 80$ in fine. Milieu $d u V I$ e siècle: Rudorff, Rechtsgeschichte, 1, § 44 . Eisele, Abhandlungen zum Civilprocess, p. 77. Après la publication du ius Aelianum (c. 554): Karlowa, Legisaktionen, 
de vingt ou trente ans au plus. Ce sera l'application d'un procédé qui n'est inconnu ni des jurisconsultes ni des historiens, mais dont les jurisconsultes n'ont peut-être point encore fait tout l'usage qu'il comporte et qui, employé avec suffisamment de suite et de précaution, permettrait, à notre avis, d'améliorer sensiblement la chronologie législative du droit privé; car c'est le seul praticable en face des lois, nombreuses surtout en droit privé, dont la tradition ne nous rapporte que le nom et les effets concrets, sans nous en faire counaître ni l'histoire externe ni les termes. On n'a, pour dater ces monuments, ni témoignages historiques ni particularités de langue et, quoi qu'en aient pensé beaucoup d'anciens copiés par trop des modernes, leur nom lui-même ne peut conduire à aucune conclusion, puisque nous sommes très loin d'avoir la liste complète des magistrats qui ont été en droit d'agir avec le peuple ou la plèbe et qu'en supposant qu'une loi donnéc doit être nécessairement de l'un des magistrats du même nom dont le hasard de la transmission nous a conservé le souvenir, on fait incorrectement abstraction de tous les homonymes ignorés qui ont pu par exemple légiférer comme préteurs ou comme tribuns. Mais rien n'empèche de chercher si la date de ces lois ne peut pas se déterminer par des consilérations de fond tirées de leurs dispositions matérielles elles-mêmes, si on ne peut pas, en prenant comme instrument ces dispositions mêmes, dégager des limites au delà desquelles

p. 348, note 3. P. Jörs, hömische Rechtswissenschaft, p. 188. Seconde moitié duVIe siècle: Bethmann-Hollweg, 2, p. 5, note 2. Cuq, Ancien droit, p. 716 (a la suite des faits qui motivèrent le sénatus-consulte de 561 et le vote de la loi Sempronia). An 577 ou 583: Ortolan, Instituts, $1^{12}, n^{\circ} .207$, suivi par beaucoup d'interprètes français: De Fresquet, 2, p. 417; Didier-Pailhé, 233, p. 339; Accarias, 24, p. 687, note 2; Appleton, Propriété prètorienne, 1, p. 22, note 2. Fin du VI siècle: Demangeat, 2 2, p. 472; Landucci, Storia del diritto romano, p. 57, note 11; Appleton, p. 34, note 9. VIe siècle: Keller, Givilprozess, \$ 23. Fin du VIe siècle ou début du VIIe: Padelletti, Storia del diritto l'ornano, pp. 251. 252. Wlassak, Processgesetze, loc. cit. An 604 environ (milieu du $I I^{\text {e }}$ siècle avant $I$. C.): Ferrini, Storia delle fonti, p. 16. Période de l'établissement des quaestiones perpetuae: Schulin, Geschichte d. rörnisch. Rechts, p. 541. Ans 605-650 entiron: Heffter, Obss. in Gai libr. IV, p. 23. Devniev siècle de la république: A. Pernice, Holtzendorff 15, 127. Entre Sex. Aelius et Cicéron ou les lois Iuliae: Bekker, $\Lambda \mathrm{ktionen,} \mathrm{1,91.}$ 
l'acte législatif à classer ne peut ni monter ni descendre, relever des moments précis où il existe nécessairement déjà et d'autres moments précis où non moins nécessairement il n'existe pas encore. La loi Aebutia, sur laquelle, en dépit de son action profonde, les seuls renseignements directs sont les textes connus de Gaius et d'Aulu-Gelle ${ }^{1}$, fournit, croyons-nous, une bonne occasion d'expérimenter cette méthode à la fois modeste et sûre. On nous excusera d'être obligé par la nature du problème à rappeler beaucoup de choses connues et à toucher seulement beaucoup de points dignes d'un examen prolongé.

I.

En ce qui concerne l'époque où la loi Aebutia existe nécessairement, le temps au dessous duquel on ne peut la faire descendre, nous avons un principe très simple et très clair. C'est la première loi qui ait dérogé au système des legis actiones. Fille est donc antérieure aux premières institutions étrangères au système que nous présentera l'histoire du droit romain. Seulement il faut discerner quelles sont ces institutions, en ayant soin de ne rien omettre de probant, en se gardant, l'autre part, de se laisser prendre à des apparences.

Ce semble, pour commencer, presque une naïveté de dire que l'existence de la procédure formulaire est attestée par les textes qui parlent de formules d'actions délivrées par le magistrat. Tout ce que l'on pourrait se demander - et c'est une question fort délicate, - ce serait s'il ne faut pas apporter à ce truisme apparent une restriction assez considérable, si les formules, ainsi d'ailleurs que la plupart des autres moyens étrangers à la procédure des Actions de la loi,

1) Gaius, 4,30: Sel istae omnes legis actiones paulatim in odium venerunt. Namque ex nimia subtilitate veterum qui tunc jura condiclerunt, eo res perducta est, ut vel qui minimum errisset litem perderet. ltaque per legem Aelutiam et duas Iulias sublatae sunt istae legis actiones effectumque est, nt per concepta verba, id est per formulas litigenus. Gell. 16, 10,8 : Sed enim cum "proletarii» et «adsidui» et «sanates» et « vales» et «subvades et. «viginti quinque asses " et "taliones" "furtorumque quaestio cum lance et licio evannerint omnisque illa duodecim tabularum antiquitas nisi in legis actionibus centumviralium causarum lege Aebutia lata consopita sit. 
n'auraient pas existé dès avant la loi Aebutia pour les procès entre pérégrins et avec pérégrins ${ }^{1}$ ). Une doctrine répandue le soutient pour les procès déférés au préteur pérégrin et, si c'était vrai d'eux, il y aurait les mêmes raisons de l'admettre pour les procès de même nature déférés avant la création de la préture pérégrine au préteur unique et aussi, croyons-nous, avant ou après, aux édiles curules. Heureusement nous pouvons nous dispenser ici d'aborder ce problème; car il n'y a, verronsnous, aucun témoignage relatif soit à la juridiction pérégrine, soit à la juridiction édilicienne qui atteste l'existence de formules - ou de moyens honoraires quelconques - avant le temps où nous en trouvons de relatifs aux citoyens attestant sans controverse possible le vote de la loi Aebutia. Nous pouvons done poser le criterium tiré de l'existence de formules sans nous occuper de la restriction hypothétique.

Un autre criterium ou plutôt plusieurs autres criteria sont fournis par le témoignage de Gaius, 4, 11, selon lequel les Actions de la loi ont été ainsi nommées, ou bien parce qu'elles étaient fondées sur des textes législatifs exprès, les édits prétoriens qui ont introduit d'autres actions n'étant pas encore en vigueur, ou bien parce qu'elles reproduisaient les termes mêmes de leur loi créatrice dont elles partageaient en conséquence l'immutabilité ${ }^{2}$ ), deux explications entre les-

1) V. de nouveau en faveur de cette doctrine, Wlassak, Processgesetze, notamment 2, p. 301 et ss. Cf. les observations d'A. Pernice, Zeitsclır. d. Sav.-Stift. 7,153. - ${ }^{2}$ ) Gaius, 4,11 : actiones, quas in usu veteres habuerunt, legis actiones appellabantur vel ideo, quod legihus proditae erant (quippe tunc edicta praetoris, quibus conplures actiones introductae sunt nondum in usu habebantur), vel ideo, quia ipsarum legum verbis accomodatae erant et ideo inmutabiles proinde atque leges observabantur. M. Wlassak, a défendu Zeitschr. d. Sav.-Stift. 9, 386. Processgesetze, 2, 304, le singulier du ms. praetoris contre le pluriel praetorum proposé par M. P. Krüger, Rechtsquell. p. 37, n. 29. Coll. jur., $2^{3}$, ad h. l., en rapportant le texte aux édits au sens étroit qui ont établi chaque action particulière. Cependant il semble que même alors Gaius eut dû plutôt employer le pluriel; car ces actions ont été introduites par les édits de plusieurs préteurs différents. En tout cas, il nous parait inadmissible de voir dans le singulier praetoris la preuve que Gaius ent voulu exclure le préteur pérégrin el ne viser que le préteur urbain; car alors il n'eut pas manqué, pour marquer l'opposition, d'ajouter le qualificatif urbani, comme il a fait par exemple, 1, 185. 
quelles il ne se prononce pas, mais qu'il considère évidemment toutes deux comme partant de faits avérés ${ }^{1}$ ). C'est l'exclusion pour la période antérieure à la loi Aebutia, soit des édits prétoriens, soit des actions prétoriennes et, malgré des dissentiments récents, il n'y a pas, croyons-nous, besoin de longs développements pour justifier ces principes généralement admis, qui sont posés en termes exprès par Gaius, entendu dans son sens le plus naturel, et qui sont conformes à tout ce que nous savons de l'évolution générale de la procédure romaine ${ }^{2}$ ). Mais le même texte implique encore à notre sens, deux exclusions moins patentes et sur lesquelles il est plus nécessaire d'insister : celle des actions civiles plus ou moins nombrenses

1) Pel importe naturellement qu'ainsi que l'a remarqué M. Mommsen, Zeitschr. d. Sav.-Stift. 12, 275, elle n'expliquent en réalité parfaitement l'expression ni l'une ni l'autre. - ${ }^{2}$ ) Cette doctrine à peu près unanime a été récemment contestée par M. Jörs, Rechtswiss. 1, 179, et par M. Wlassak, Processgesetze, 2, 303 ss. Zur Gesch. d. Cognitur, 59 ss. M. Wlassak, qui est celui dont l'argumentation est la plus pressante et qui seul discute le texte fondamental de Gaius, essaie de l'écarter en rapportant le mot tunc non pas au temps dans lequel les Actions de la loi étaient en usage ou étaient ainsi nommées, mais à celui de leur création. Il s'entend cependant, en grammaire et en raison, un pell mieux de l'époque de leur application, de leur application exclusive, dirons-nous avec le système de M. Wlassak que cela ne contredit aucunement quant au point qui nous intéresse. La meilleure preuve en est qu'on l'avait toujours compris ainsi. M. Wlassak lui-même n'aurait probablement jamais songé à son interprétation, si elle n'était nécessaire pour admettre, au temps des Actions de la loi, ce libre exercice fait, en matière judiciaire, de l'imperium qui, disent lui et M. Jörs, existait aussi bien avant la loi Aebutia qu'après elle. Mais là encore il y a une confusion. Sans doute l'imperium est aussi. vieux que la magistrature. Seulement il s'agit de savoir comment le magistrat doit l'exercer, s'il peut en user à sa guise ou s'il doit le faire conformément à des lois. Or Gaius, entendu dans son sens naturel, affirme qu'en matière de procédure l'exercice en était, à l'époque ancienne rigoureusement règlé par la loi, et c'est aussi la tradition romaine; car, M. Wlassak le remarque quelque part, elle considère déjà l'imperium royal comme un imperium legitimum et surtout elle représente la loi des XII tables comme faite pour soustraire l'administration de la justice aux incertitudes résultant du défaut de lois écrites et d'ailleurs un peu exagérées par certains textes (cf. Pomponius, D. 1, 2, 2, 1.3.4) en vertu de l'idée grossière qu'il no pourrait $\mathrm{y}$ avoir de droit établi sans législateur individuel. 
qui, à côté de leur formule in ius, ont une formule in factum et celle des actions de bonne foi.

Quant aux premières, on a pa essayer d'expliquer la dualité par tel ou tel avantage de la formule in factum, tant qu'on a cru y voir une singularité accidentelle du dépôt et du commodat seuls visés par Gaius, 4,47. La situation a changé depuis que l'on sait que les deux formules se rencontrent non seulement là, mais certainement en matière de gage où on le soupçonnait un peu, probablement en matière de mandat et même de gestion d'affaires où nul ne s'en doutait, peut-être en matière de fiducie où cela renverserait toutes les idées antérieurement reçues; depuis que l'on sait en même temps que, tout au moins dans les cas de commodat, de gage et de dépôt, c'est la formule in factum qui occupe le premier plan dans l'album et dans les commentaires. On ne peut plus voir désormais dans cette formule un instrument exceptionnel employé en des cas particuliers pour satisfaire à quelque besoin incident. Elle ne peut plus s'expliquer que comme la formation première à laquelle est venue ensuite se juxtaposer à titre auxiliaire et en vue d'avantages propres la formule in ius '. Et alors toute action présentant les deux formules implique la préexistence de la loi Aebutia après laquelle seule

1) L'idée que la dualité de formules suppose l'antériorité de la formule in factum a été depuis longtemps exprimée (v. Desjardins, R. hist. de dr. français et étranger, 1867, p. 122 ss.; Ubbelohde, Zur Gesch. đ. benannt. Realcontracte, p. 62 ss. Wlassak, Neg. gest. p. 152 ss.). Elle est, croyons-nous, rendue indéniable par la connaissance nouvelle de l'édit que nous devons aux travaux de Lenel. M. Lenel paraît lui-même l'indiquer en un on deux endroits, mais pourtant il ne l'a pas affirmée expliciternent et il reculerait peut-être devant son application à certaines actions, par exemple à l'actionl fiduciae, de même qu'il n'admet pas l'action in factum dans tous les cas que nous avons cités, notamment dans celui de negotiorum gestio. Nous renvoyons à son ouvrage, Ed. \$§ 98. 99. 106, pour le classement des deux formules de dépôt, de commodat et de gage; $\$ 108$, p. 236, pour l'existence d'une formule in factum au cas de mandat; $\$ 107$, p. 233 , pour l'existence et la prépondérance de la formule in factum au cas de fiducie. V. cependant, quant à cette dernière, Pernice, Labeo, 3, 1, p. 124. Karlowa, Röm. Rechtsgesch. 2, 2, p. 561. L'existence, au moins à l'origine, d'une formule prétorienne en matière de gestion d'affaires nons semble, comme à M. Wlassak, Nag. gest. p. 21 et ss., prouvée par l'édit D. 3, 5, 3, pr. 
a pu naître la formule prétorienne in factum suivie à son tour par la formule in ius.

Il en est de même, à mon sens, de toutes les autres actions de bonne foi; en effet il y a un texte formel qui implique qu'au moins en tant qu'actions de bonne foi elles datent seulement de la procélure formulaire. C'est le passage de Cicéron, de officiis, 3, 15, 61, selon lequel dolus vindicatur ... sine lege iudiciis in quibus additur ex fide bona ${ }^{1}$ ). Il implique que les actions de bonne foi sont postérieures à la loi Aebutia, s'il signifie, comme ont pensé plusieurs, que les iudicia dont il parle existent sine lege. Mais il l'implique aussi, en réalité, s'il signifie, comme ont objecté l'autres, que le dol y est réprimé sine lege en vertu de la clause ex fide bona; car alors la clause n'est pas ipsius verbis legis accommodata et par conséquent elle ne peut exister que depuis la procédure formulaire. Interprété dans le premier sens, le texte prouve qu'il n'y avait pas d'actions de honne foi sous les Actions de la loi, à cause de la première explication de Gaius; interprété dans le second, il le prouve à cause de la seconde ${ }^{2}$ ). Tout ce que l'on pourrait admettre, - et c'est uniquement pour cela que nous avons mis à part les actions à deux formules qui ne comportent pas la même réserve, - ce serait que les actions qui furent plus tarl de bonne foi et pour lesquelles il n'y a pas de vestiges de formule in factum, auraient existé dès alors sans la clause ex fide bona, par exemple en matière de vente et de louage, comme iudicia stricti iuris ${ }^{3}$ ).

1) De off. 3, 15, 61 : atqui iste dolus malus et legibus erat rindicatus, ut in (in manque dans les mss.) tutela duodecim tabulis, circumscriptio adulescentium lege Platoria, et sine lege iudiciis, in quibus additur ex fide bona. - ${ }^{2}$ ) Le texte est entendn dans le premier seus par Dernburg, Comp². p. 59, n. 3; Kuntze, Krit. Vierteljahrschr. 9, 519; Karlowa, Legisaction. p. 128; Krüger Rechtsquell. p. 44, n. 37. L'ohjection tirée à tort de la seconde interprètation a été formulée par Arndts, Gesammt. Schr. 1, p. 403 ss. et Ubbelohde, Zur Gesch. d. Realcontr. p. 81. $-{ }^{8}$ ) V. dans ce sens Bechmann, Kauf, 1, 467. 472. 475. Burckhardt, Zur Gesch. d. locatio, p. 13. 56. Hugo Kŕ̈ger, Zeitschr. d. Sav.-Stift. 11, 193. Cette conception se concilierait tout particulièrement avec la doctrine de M. Baron, Condictionen, p. 195 ss. selon laquelle la condictio aurait été un instrument universel apte à faire valoir toutes les prétentions reconnues obligatoires. Mais voir contre cette doctrine les objections graves de Pernice, Labeo, 3, 1, 228. 
Un autre terme d'exclusion est fourni, d'après Gaius, 4, 108, par les textes attestant l'existence de ces exceptions, et, peuton dire, aussi de ces prescriptions, dont l'usage n'existait pas au temps des Actions de la loi comme du sien ${ }^{1}$ ), à condition bien entendu de savoir distinguer la réalité des apparences et de ne pas voir la preuve d'exceptions dans tous les témoignages relatifs à des droits qu'elles ont plus tard sanctionnés.

Il est enfin un dernier criterium qui ne s'appuie pas sur des témoignages directs, mais dont il ne nous semble pas possible de faire abstraction. C'est celui tiré du droit du magistrat d'admettre ou de repousser la demande, de dare ou de denegare legis actionem, dit-on en visant la procédure des Actions de la loi. Beaucoup d'auteurs almettent que le magistrat aurait eu ce pouvoir dès le temps de la domination exclusive des Actions de la loi et on s'en sert même souvent pour faire remonter à cette période des moyens sanctionnés par des exceptions à l'époque postérieure ${ }^{2}$ ). A notre sens, il n'est rien de plus contraire à la notion vraie de la procédure primitive, où le magistrat n'a pas plus à donner

1) Gaius, 4, 108 : nec omnino ita, ut nunc, usus erat illis temporibus (legis actionum) exceptionum. - ${ }^{2}$ ) Le système connu, selon lequel les exceptions futures auraient alors été remplacées par des sponsiones à la suite desquelles le magistral accordait on refusait la legis actio, part de cetle idée. Mais nons repoussons également la doctrine de ceux qui, tont en niant les sponsiones, admettent que le magistrat peut, après examen, empêcleer la legis actio en lui refusant son concours (Karlowa, Legisactionen, p. 343; Lenel, Ursprung der Except. 41 ss.). Parmi les textes invoqués qui impliquent réellement le pouvoir arbitraire du préteur, il n'y en a, verrons-nous, aucun qui se place avant la période où la procédure formulaire peut exister (Huschke [Multa, p. 496, n. 400] ne les invoque que dans ce sens). Quant au cas des vites de Gaius, 4, 11, dont parle M. Karlowa, Gaius ne dit pas que l'accomplissement matériel d'une seconde legis actio devant le magistrat eut été impossible; ce serait, pensons-nous, le juge qui ent reconnu son mal foudé, et il en est de même d'à peu près toutes les hypothèses où MM. Karlowa et Lenel supposent un examen préalable du magistrat. M. Lenel fait remarquer qu'au cas d'action réelle on ne voit pas ce qui dans les paroles sacramentelles soumettrait au juge la question de la déduction du droit en justice. Mais précisément le cas d'action réelle est, à côté de cas appartenant à la procédure moderne, le seul où le droit ne soit pas éteint ipso iure (Gaius, 4, 107). C'est peut-être la preuve que la règle bis de eadem re ne s'y est pas appliquée dès le principe. 
l'action qu'à la refuser, où tout son rôle se résume dans un concours, pour ainsi dire mécanique, à la solennité légale, où il commettrait également une forfaiture en refusant ce concours dans les conditions légales et en essayant de lui faire produire effet hors des conditions légales. C'est seulement quand la loi Aebutia eut mis à côté de la procédure ancienne la procédure nouvelle, eut établi entre les deux un choix soumis à son contrôle ${ }^{1}$ ) que le magistrat acquit et dut nécessairement acquérir non pas seulement la possibilité de fait, mais la possibilité de droit, de refuser son concours à la procédure ancienne, de le refuser par exemple quand il ne voulait le donner qu'à la procédure nouvelle. Le droit de denegare legis actionem est un corrélatif inséparable de celui de délivrer la formule. Il n'y a pas d'effet de la loi Aebutia qui ait dâ en résulter plus immédiatement ${ }^{2}$ ). Mais c'est un effet

1) C'est là, à notre sens, le point essentiel. Il y a peut-être eu auparavant des cas où le demandeur pouvait choisir entre deux legis actiones, comme on l'a soutenu, soit en invoquant Gaius, 4, 20, pour la iudicis postulatio (Wlassak, Processgesetze, 1, 105; A. Schmidt, Zeitschr. d. Sav.Stift. 2, 150 ss. Gradenwitz, id. 9, 191), soit, avec encore plus de raisons, croyons-nous, pour la condictio, en invoquant Gaius, 4, 95 (Wlassak, Processgesetze, 1, 106). Mais rien n'indique là que le choix émane d'un autre que du demandeur, maitre de son action qu'il dirige comme il veut, et par suite aucune intervention du magistrat n'est nécessaire afin de lui refuser une legis actio après qu'il a pris l'antre; la règle bis de eadem re suffit. Au contraire il faut un pouvoir du magistrat qui force le demandeur à changer de voie, quand le choix de la procédure n'est plus laissé à son initiative, quand il dépend du défendeur comme pour la procédure sine periculo des interdits (Gajus, 4, 163 ss.), ou, comme c'est plut ôtle cas dans notre matière, soit du magistrat seul, soit du défendeur assisté par le magistrat. Or M. Wlassak a très bien vu que le concours électif de l'ancienne procédure et de la nouvelle ne peut avoir été laissé à la seule volonté du demandeur, qu'il doit avoir dépendu en dehors de lui, de celle du magistrat, soit au cas de dissentiment, soit peut-être sans cela. Il s'est seulement trompé, croyonsnous, en prenant pour l'instrument préexistant de la réforme un pouvoir du magistrat qui en a senlement été la conséquence. - ${ }^{2}$ ) V. la note précédente. C'est même, croyons-nous, là qu'il faut chercher la source des pouvoirs nouveaux accordés au magistrat depuis la loi Aebutia, qui n'ont pu lui venir que d'une loi positive et qui, d'autre part, ne peuvent guère lui avoir été accordés par une abdication consciente du pouvoir législatif. Pour forcer le demandeur à agì per formulas, il a fallu nécessairement permettre au magistrat de refuser son concours à la 
de la loi Aebutia qui n'a pu exister avant elle. Nous jugerions par conséquent très hasardé de considérer .la procédure des Actions de la loi comme encore seule en vigueur à un moment où on trouverait la preuve du droit du magistrat d'empêcher l'accomplissement de la legis actio ${ }^{1}$ ).

Formules, édits prétoriens, actions prétoriennes, actions à double rédaction in ius et in factum, actions de bonne.

legis actio. Pour le forcer à agir per legis actionem, il a fallu permettre au magistrat de lui refuser la formule. C'était l'instrument indispensable du nouveau système. Et on n'en a probablement pas vu davantage en faisant la réforme. Mais, par là même que la concession des legis actiones et des formules dépendait désormais du magistrat, on se trouvait l'avoir investi, d'une façon plus inconsciente que calculée, d'un pouvoir tout nouveau sur les procès; on lui avait onvert la voie des réformes législatives en lui permettant d'étouffer les prétentions fondées sur la loi lorsqu'il refuserait la legis actio sans délivrer de formules corrélatives, de sanctionner des prétentions sans bases légales lorsqu'il délivrerait des formules d'actions ne correspondant qu'en partie ou ne correspondant pas du tout aux lois antérieures. Et c'est, à notre avis, par cette fissure que le préteur, appuyé sur l'opinion publique et les jurisconsultes, a pu faire pénétrer dans le domaine du droit toutes les prétentions juridiques nées de conceptions et de besoins nowveaux anxquelles l'ancien système refusait une satisfaction légale.

1) Nons négligeons d'antres criteria comme plus incertains. Ainsi la prohibition de lege agere alieno nomine ne peut servir d'instrument à cause de l'exception praeterquam ex certis crusis de Gains, 4, 82. Ainsi, fût-elle admise, la conjecture favorite de nos anciens auteurs, reprise par M. Wlassak, Processgesetze, 2, 291, n. 19, selon laquelle le tribunal des centumvirs aurait élé créé par la loi Aebutia, ne mènerait à ancun résultat, parce que le premier témoignage relatif à la justice centumvirale se rapporte seulement à l'orateur L. Crassus, mort en 663 (Brut. c. 39, 145. c. 52). Un terme plus précis serait faurni par les décemvirs litibus iudicandis, si on admettait, comme on ne l'a pas encore fait à notre connaissance, mais comme il serait dans la logique du système, qu'ils aient été créés par la mème loi; car alors on aurait une date fixe et pratique, tirée du décemvirat occupé an déhut de sa carrière par Cn. Cornelins Scipio Hispanus préteur pérégrin en 615 (Val. Max. 1, 3, 2) : il avait été auparavant édile et questeur (C. I. L. I, n. 38) et il doit donc, en tenant compte de l'intervalle de la loi Villia, avoir été décemvir en 608 au plus tôt, quelques années même auparavant si l'on suppose ses deux tribunats militaires postérieur's au decemvirat ainsi que semble indiquer son inscription. Mais le raisonnement serait bien hypothètique et la date nous paraitrait d'une précocité excessive, en face des lois Calpurnia et Iunia dont nous parlons plus loin. 
foi, exceptions, dénégation par le magistrat de la legis actio: cela fait en somme jusqu'à sept indices d'ordre distinct dont aucun, croyons-nous, ne pourrait être relevé sans accuser l'existence de la loi Aebutia. Il n'en est que plus frappant de constater qu'on les cherche vainement tous jusqu'aux environs de l'an 630 pour les voir ensuite tous brusquement apparaître à partir de là. Nous jugeons superflu de remonter au delà de l'an 500 de Rome. Mais il est indispensable de passer en revue les textes relatifs au $\mathrm{VI}^{\mathrm{e}}$ siècle et au début du VII e.

Pour le $\mathrm{VI}^{*}$ siècle, nous nous heurtons immédiatement à une controverse connue. On a, non pas pour notre question, mais pour celle de l'antiquité des contrats de bonne foi et particulièrement des contrats consensuels, prétendu découvrir des vestiges de la vente et du louage classiques dans le théatre de Plaute, mort en 570, dans l'un des rares témoignages qui nous soient parvenus de l'activité doctrinale du jurisconsulte Sex. Aelius Paetus Catus, cos. en 556, censeur en 560, et enfin dans le traité d'économie rurale écrit durant les dernières années de sa vie par Caton l'Ancien, mort en 605. Mais, en nous bornant à notre question propre, aucun de ces documents ne fournit pour elle d'argument topique. Les ventes du théâtre de Plaute, sur lesquelles la polémique a été particulièrement vive, sont, on paraît tendre à le reconnaître, un mauvais terrain de discussion, parce que ce théâtre est copié sur des modèles grecs, que l'intrigue des pièces y est prise à la Grèce et que, si l'auteur y plaque, afin de piquer l'attention des spectateurs, des détails de procédure romaine, des termes techniques romains, on ne peut jamais aftirmer que le fond soit romain ${ }^{1}$ ). En tout cas, si l'on prétendait y trouver la preuve de contrats consensuels, il n'y aurait aucun indice que ces contrats fussent de bonne foi ${ }^{2}$ ),

1) Cf. P. Krüger, Rechtsquell. pp. 76. 77. V. les exernples de Bechmann, Kauf, 1, 504 et ss. Burckhardt, Locatio, p. 6 et ss. - ${ }^{2}$ ) Il nous est impossible d'insister sur cetle controverse où l'incertitude de l'instrument a conduit aux solutions les plus opposées, les uns concluant avec M. Bekker, Aktionen, 1, 511 et ouvrages antérieurs (v. aujourd'hui Zeitschr. d. Sav.-Stift. 13, 109 ss.), que la vente consensuelle était inconmue au temps de Plaute, les autres avec Demelius, Zeitschr. 
et cela suffirait pour les rendre étrangers à nos recherches. La même observation permet d'écarter du débat les formulaires si intéressants pour l'histoire de l'ancienne pratique romaine que donne Caton des marchés agricoles. On a prétendu voir dans leur chapelet de clauses successives des modèles de contrats consensuels de vente et de louage. On pourrait au moins aussi bien y reconma1tre des accumulations de clauses divergentes rendues en bloc obligatoires par une stipulation finale. Mais rien n'indique qu'ils fassent naître des actions de bonne foi. Leur rédaction prudente et compliquée, dans laquelle les parties se croient forcées de tout spécifier et de tout prévoir, implique même l'idée contraire ${ }^{1}$ ). - Le document le plus sérieux serait celui qu'on a cru trouver dans la citation de Sex. Aelius Paetus Catus faite par Celse, D. 19, 1, 38, 1. Celse rapporte que Drusus et Sex. Aelius admettaient en cas de retard dans la déliviance imputable à l'acheteur pro cibariis per arbitrium indemnitatem posse servari. C'est, a-t-on dit, la preuve que l'arbitrium venditi, l'action de bonne foi du vendeur existe dès le VI ${ }^{\theta}$ siècle. Mais il y a une considération qui rend cet argument facile à écarter. C'est que, verronsnous, Sex. Aelius est précisément invoqué à bon droit pour prouver l'inexistence de la loi Aebutia de son temps. Il faut donc encore admettre là ou qu'il s'agit d'une action venditi de droit strict antérieure à la loi Aebutia et d'ailleurs peu probable à notre sens, ou que le témoignage est mal compris, soit que, comme a dit M. Bekker, il se rapporte à la stipulation pro cibariis arbitrio boni viri indemnitatem servari faite entre le vendeur et l'acheteur, soit plus largement que Celse et peutêtre déjà Drusus aient modernisé en le rapportant à la vente

f. RG. 2, 177 ss. Karlowa, Legisactionen, 138 ss. Costa, Dir. prival. nell. comm. di Plauto, 365 ss., qu'elle existait déjà comme contrat consensuel et de bonne foi sauf de la part du dernier un peu d'hésitation sur le second point, d'autres, comme Bechmann, loc. cit. qu'elle existait comme contrat consensuel, mais non comme contrat de bonne foi, ce qui nous paraitrait le terme extrème des concessions possibles.

1) La même polémique se continue jusqu' à un certain point sur les formulaires de Caton, qui nous semblent la preuve directe que ces contrats n'étaient pas encore reconnus, au moins comme contrats de bonne foi, à l'époque de Caton ni par conséquent à celle de Plaute. Cf. Bekker, Zeitschr. für RG. 3, 416 et ss. Karlowa, 133 et ss. Bechmann, 537. 618. 
du droit nouveau ce que Sex. Aelius disait des contrats verbaux conclus entre le vendeur et l'acheteur ${ }^{1}$ ). Le texte se trouve tout au moins être ainsi lui-même étranger à notre question. La période du VI ${ }^{\circ}$ siècle n'offire pas plus de vestiges $\mathrm{n} 1$ du droit du magistrat d'accorder ou de refuser l'action, ni des exceptions dont ce droit a été la condition préalable, ni des formules au sens technique, telle qu'est par exemple la formule pétitoire des actions réelles ${ }^{2}$ ), ni des actions prétoriennes, comme est l'action publicienne ${ }^{3}$ ), comme sont encore, sans

1) V. Bekker, Aktion. 1, 314. Bechmann, 1. 637. H. Krüger, Zeitschr. d. Sav.-St. 11, 195. Cf. en sens contraire Karlowa, 132 et ss. $\left.{ }^{2}\right)$ L'existence de la formule pétitoire est, à notre sens, établie en matière de revendication, vers la fin du VII e siècle, par Cicéron, In Verr. 2, 13, 31. Cf. la démonstration développée de Wlassak, Processgesetze, 1, 115 et ss. et P. Krüger, Krit. Vierteljahrschr. N. F. 13, 326. Majs son caractère récent nous semble résulter de ce qu'elle n'est pas encore étendue à la pétition d'hérédité pour laquelle le même Cicéron, In Verr. 1, 45, 115, ne connaît que la legis actio et la procédure per sponsionem. Cf. Wlassak, 1, 112. - ${ }^{3}$ ) L'action publicienne n'étant qu'un remaniement de la formule pétitoire de la revendication ne peut pas être plus ancienne qu'elle. Il n'y a, en dehors de là, aucun témoignage sur son introduction. Nous négligeons naturellement les systèmes qui ne se basent que sur des rencontres de noms propres et des affirmations gratuites, ainsi celui de M. Voigt, Ius naturale, 4, p. 504 et ss. qui admet que l'action vient nécessairement ou de M. Publicius Malleolus, cos. en 522, dont il place la préture en 519 (à cause du bien. nium exigé cinquante ans plus tard par la loi Villia?) ou de Q. Publicius, prèteur en 687 d'après Cicéron Pro Cluent. 45, 126, et qui se prononce en faveur du premier pour déduire ensuite de là que la loj Aebutia aurait été rendue auparavant. S'il fallait choisir, nous préférerions le Publicius de 687. Mais, bien que le nombre des personnages qui ont pu rendre des édits comme préteurs urbains ou pérégrins soit sensiblement inférieur au nombre de ceux qui ont pu faire des lois et que l'argument tiré de la similitude des noms soit done là un peu moins faible, il est encore, au moins à lui seul, inacceptable, parce que nous n'avons pas la liste complète des préteurs. Quant au Publicius de Cicéron, il se trouve être certain qu'il n'a pas été le créateur de l'action publicienne; car cette action fondée sur la fiction de l'usucapion n'a pu être créée que pour les citoyens par un préleur urbain; or, en 687 , le préteur urbain n'était pas Q. Publicius, mais l'autre préteur cité par Cicéron, M. Iunius. Cela résulte déjà, comme l'a remarqué M. Pernice, Labeo, 2,158 , n. 19, de ce que Cicéron le cite le premier (cum defendissem apud M. Iunium Q. Publicium praetores); cela résulte en outre de ce qu'il a suivant Pline, H. n. 35, 36, 100, donné les jeux Apollinaires. V. les Fasti 
parler des actions nées de délits dont la création se place à une date postérieure certaine, les actions furti manifesti au quadruple $^{1}$ ) et iniuriarum aestimatoria. On a bien prétendu, plus au cours de recherches spéciales indépendantes qu'en vue de notre problème, discerner çà et là des apparitions isolées de tel ou tel de ces moyens. Mais il n'y en a pas, suivant nous, une seule qui soit scientifiquement avérée. Ainsi nous citerons l'allusion à l'action d'injure estimatoire qu'on a relevée en l'an 560, chez Plaute dans l'asinaria, 2, 2, 104. Les mots pugno malam si tibi percussero sont, a-t-on dit ${ }^{2}$, visiblement empruntés à la formule de l'action prétorienne donnée Coll. 2, 6, $4:$ quod $A^{\mathrm{i}} A^{\mathrm{i}}$ mala pugno percussa est et il $\mathrm{y}$ a en effet une concordance de termes très frappante. Elle cesse d'être bien étonnante si l'on réfléchit que longtemps avant l'édit il y a eu des poings qui sont tombés sur des visages et qui y sont même tombés contrairement à la loi des XII tables, et que la formule concrète d'action soumise comme modèle aux plaideurs a dâ précisément être choisie parni les variétés d'injures les plus usuelles. C'est sous le régime des XII tables, avant l'action prétorienne, que la tradition représente le proverbial Veratius distribuant à droite et à gauche ses soufflets tarifés ${ }^{3}$ ); rien n'empêche que Plaute pense aussi pour la répression des siens à l'amende invariable des XII tables si tant est qu'il songe à une loi romaine quelconque ${ }^{4}$ ). -

praetorii de Wehrmann, p. 48 et de Hölzl, p. 31. Publicius ne parait mème pas avoil occupé la préture pérégrine : cf. Hölzl, p. 29. Quant aux combinaisons par lesquelles Huschke, Publ. Klage, 115 ss. a essayé de donner une certaine antiquité à la publicienne, nous les croyons avec M. Pernice, loc. cit., déuuées de valeur.

1) M. Costa renonce à trouver dans le théâtre de Plaute un vestige de l'action furti manifesti au quadruple. - ") Lenel, Edictum p. 321, note 4. Cf. Huschke, Gaius p. 128. Voigt, Röm. Rechtsgesch. 1, 704, n. 28. M. Voigt remarque d'ailleurs, ainsi qu'il est rationnel quand on traite les textes de Plaute en textes juridiques, que dans Plaute le sujet actif de l'action est un pérégrin et n'en prouve donc l'existence que dans l'édit du préteur pérégrin. $-{ }^{3}$ ) Gell. 20, 1, 13. Cf. Rudorff, Ed. p. 175, n. 8, oú il explique la formule par le cas de Veratius. - ${ }^{4}$ ) Un témoignage infiniment plus sérieux a paru un mornent fourni par le sénatus-consulte de Thisbaeis de l'an 584, dans lequel les premiers éditeurs avaient cru trouver une mention de l'action d'injure estimatoire, au reste au profit de pérégrins 
Quant aux exceptions, il est à peine besoin de réfuter aujourd'hui la doctrine ancienne ${ }^{1}$ ) selon laquelle les exceptions fondées à l'époque des jurisconsultes classiques par les lois Cincia et Plaetoria prouveraient l'existence de la loi Aebutia à l'époque de ces lois qui sont du milieu du VI e siècle, la loi Cincia de 550 et la loi Plaetoria des environs de $562^{2}$ ). Rien n'exige que les lois Cincia et Plaetoria aient été dès le principe sanctionnées par des exceptions. Elles peuvent l'avoir été auparavant par des moyens différents. Nous en avons la preuve positive pour la loi Plaetoria dont l'action civile, que l'exception a plus tard remplacée par simplitication, est attestée par des textes formels et on peut admettre la même solution pour la loi Cincia qui est la plus ancienne ${ }^{3}$ ). Si donc ces lois prouvent

et devant le préteur pérégrin. Mais il n'y avait là qu'une fausse lecture qui a depuis longtemps disparu du texte définitif du monument (Bruns fontes ${ }^{5} 152$ et ss.) et que nous relevons uniquement parce qu'elle a passé dans quelques ouvrages juridiques (Dernburg, Festgabe für Heffter, p. 101 ; de Ihering Jahrbücher, 23, 155; Willems, sénat, 2, 277, n. 1). Le texte de Caton, p. 53, 2 (Jordan), invoqué à la fiı du VIe sjècle par Voigt ne prouve rien. Les autres textes cités par le mème auteur se rapportent à des dates certainement postérieures à celle où nous admettons l'existence de la loi Aebutia.

1) Puchta, Inst. 1, $\S 80$ in fine. $-{ }^{2}$ ) La loi Cincia a été votée, comme on seat, en l'an 550, sous le consulat de Tuditanus et de Cethegus, selon Cicéron, Cato maior, 4, 10. Quant à la loi Plaetoria, les allusions certaines du Pseudulus (1, 3, 69. 70 : perii : annorum lex me perdidit quina vicenaria : metuont credere omnes) et du Rudens (5, 3, 24-26: cedo quicum habeam iudicem ni dolo malo instipulatus sis nive etiain dum siem minus quinque et viginti natus annos) représentés le premier en 563 et le second vers la mème époque (Teuffel, $1^{5}, \S 97$ ) prouvent qu'elle existait el qu'elle existait depuis peu à cette date. D'autres pièces du mène Plaute paraissent à M. Costa, Bull. dell. ist. 2, 72 ss. Dir. privat. 200-205, impliquer qu'elle n'existait pas à leur époque et permettre de la placer entre 560 et 562 , dans la seconde moitié de 561 ou la première de 562. - ${ }^{3}$ ) On connaît pour la loi Plaetorja la démonstration lumineuse de M. de Ihering, Geist, 3, 1+, 119. M. de Ihering a seulement eu tort, croyons-nous, de conclure à l'existence d'une action unique, tandis que, selon la juste observation de M. Karlowa, Legisactionen, 352-353, la loi Iulia municipalis, lignes $110-112$, prouve positivenent l'existence de deux actions dont l'une est le iudicium publicum de Cicéron et l'autre l'action plus tard remplacée par l'exception. C'est encore à cette dernière action que se rapportent, sans grand doute possible, le $f r$ de formula Fabiana, 4, qui la qualifie de noxale et le 
quelque chose, c'est que, du temps de la plus récente, le résultat plus tard atteint par une exception a encore, été poursuivi par un procédé différent. Il faut un peu plus insister sur une trace de l'exception de dol qu'on a cru trouver dans un texte de Plaute qui cette fois s'occupe indubitablement de droit romain, dans un texte du Rudens, 5, 3, 24 et ss. que nous venons de mentionner au sujet de la loi Plaetoria et dans lequel un personnage dit à un autre : cedo quicum habeam indicem ni dolo malo instipulatus sis nive etiam dum siem minus quinque et viginti natus annos. Ces vers supposent, a-t-on dit ${ }^{1}$ ), au profit de l'obligé, soit une exception, soit un moyen équivalent fondé sur le dol à côté de celui fondé sur la loi Plaetoria; or, s'il s'y agissait véritablement d'une défense tirée du dol, nous admettrions que cela prouverait l'existence de la loi Aebutia, qu'il s'agit de l'exception en forme ou d'une de ces sponsiones conjecturales qui sont à notre avis également incompatibles avec la domination exclusive de l'ancienne procédure. Mais, quelque répandue que soit l'interprétation qui voit ici la mention distincte de deux moyens, elle n'en est pas moins en contradiction flagrante avec le système de la loi Plaetoria selon lequel il doit s'agir non pas de deux moyens fondés sur des causes différentes, le dol et la minorité, mais d'un moyen unique, celui de la loi Plaetoria, dont l'admission exige deux conditions distinctes : la minorité de la victime et le dol du cocontractant. Le texte les cite correctement toutes deux et le personnage qui parle se déclare prêt à plaider sur le double point de savoir si 1) son adversaire n'a pas usé de dol et 2) si lui-même n'était pas en état de minorité. L'une des preuves à elle seule serait aussi impuissante que l'autre. Pour la loi Plaetoria, cela résulte de principes élémentaires. Et pour le dol ce serait prouvé, s'il en était besoin, par le texte de Cicéron, de officiis ${ }^{2}$ ), qui, si torturé qu'il ait été dans

texte de Paul, D. 44, 24,3 (v. mes observations Nouv. Rev. hist. de droit, 1890, pp. 696-700). Pour la loi Cincia, v. Karlowa, pp. 348-352.

1) Burchardi, Wiedereinsetzung, p. 300 ss. D'autres, comme A. Pernice, Labeo, 2, 96, n. 6, admettent l'existence du moyen, mais le rapportent à la procédure des Actions de la loi. $-{ }^{2}$ ) Cicéron, de off. 3, 14, 58-60: emit homo cupidus et locuples tanti, quanti Pythius voluit, et emit instructos; nomina facit, negotium conficit... stomachari Canius; 
tous les sens, reste toujours l'affirmation positive qu'avant Aquilius Gallus, il n'y avait pas de ressource légale pour celui qui s'était engagé sous l'empire du dol, par conséquent pas

sed quid faceret? nondum enim C. Aquilius, collega et familiaris meus, protulerat de dolo formulas. Les deux autres texles qu'on invoque parfois afin d'établir l'existence de l'exception de dol avant Aquilius Gallus, celui relatif à l'exception donnée contre les publicains par Q. Mucius Scaevola dans son Gouvernement d'Asie (Ad Att. 6, 1, 15) et celui d'Ulpien, D. 44, 4, 4, 33, selon lequel metus causa exceptionem Cassius non proposuerat contentus doli exceptione quae est generalis, sont faciles à écarter. L'exception établie par Q. Mucius Scaevola n'a certainement pas été, d'après tout ce que nous savons de Q. Mucius, de l'intégrité de son administration et du mécontentement qu'en éprouvèrent les chevaliers (Cicéron, ad fam. 1, 9, 26; pro Plancio, 13, 33 ; cf. Mornmsen, Röm. Gesch. $2^{8}, 211$ ), une édition atténuée au profit des publicains d'une exception déjà donnée plus sévèrement contre toul le monde. Elle a élé une première application, restreinte soit à certains dols, soit aux dols de certaines personnes, d'une protection plus tard accordée contre tous. Ce qu'elle prouve donc, ce n'est pas l'existence, c'est l'inexistence de l'exception de dol ordinaire. Au contraire le texte d'Ulpien, D. 44, 4, 4, 33, prouve à notre avis l'existence de l'exception de dol et mème de l'exception metus; car', si la seconde n'avait pas déjà existé, Cassius n'aurait pas en grand mérite à ne pas la proposer, c'est à dire en somme à ne pas l'inventer. II se place donc au plus tard à l'époque de la création de la dernière créée des deux qui serait alors l'exception de dol, mais heaucoup plus probablement à un mornent où elles existaient déjà depuis un temps plus ou moins long et où le préteur dont il s'agit les trouvait l'une et l'autre dans les édits de ses prédécesseurs; car c'est d'un préteur qui, trouvant suffisante une exception existante, ne propose pas une autre exception existante que le texte s'entend le mieux. Mais ce préteur Cassius n'est, croyons-nous, ni le consul de 667, auquel ont pensé beaucoup d'anciens interprètes, ni le consul de 630 (Voigt, Ius nat. 3, 905), ni celui de 681 (Rudorff, Ed. p. 55), ni celui de 647 ou celui de 681 (Huschke, Zeitschr. f. Civilr. und Process, 14, 127) ni un préteur antérieur à 684 (Pernice, Labeo 2, 96). C'est, nous semble-t-il évident, quoique personne ne paraisse y songer, le seul personnage dont parlent les jurisconsultes quand ils disent Cassius tout court (v. Krüger, Rechtsquell. 154, n. 50) le jurisconsulte Cassius, Gaius Cassius Longinus, consul en l'an 30 après J.-C. et par conséquent prèteur avant l'an 28, que d'autres textes montrent avoir occupé l'une des deux prétures civiles, assez probablement la préture urbaine, et avoir, conformément à son caractère et à ses traditions de famille, imité les magistrats jurisconsultes de la République en faisant comme eux des additions et des remaniements it son édit. Voir par ex. D. 4,6, 26, 7 : Gaius Cassius nominatim 
plus d'exception au sens propre on au sens large que d'action ou de restitutio in integrum. L'exception de dol ne peut pas être dans Plaute au VI ${ }^{\text {e }}$ siècle; car l'action de dol a seulement été créée à la fin du VII ${ }^{*}$ par Aquilius Gallus ${ }^{1}$ ) et l'exception n'a pas existé avant l'action, qu'elle ait d'ailleur's été créée en niême temps qu'elle, comme l'ont pensé plusieurs auteurs anciens ${ }^{2}$ ) ou que, suivant un ordre de succession qui est aujourdhui établi pour l'action et l'exception metus $^{3}$ ) et qui me semble parfaitement d'accord avec la marche générale du droit prétorien, l'exception ait ici encore été introduite un peu après l'action. - Nous aurions aussi peine à concevoir l'existence d'un chef quelconque de restitutio in integrum avant la loi Aebutia, qui seule put permettre au magistrat de déduire de la restitutio des conséquences pratiques, de donner les actions refusées par le droit civil ou de paralyser d'une manière quelconque celles accordées par lui. Mais nous jugeons superflu d'insister sur la restitutio ob absentiam (demandée par un père contre les actes d'un fils de famille!) qu'on a parfois cru trouver jadis dans le Phormio, avant l'an 595, date de la mort de Térence et même l'an 593, date probable de la première représentation de la pièce, et

edicebat. D. 29, 2, 99: sanctum (Cujas corrige Gaium) Cassium praetorem causa cognita actiones utiles daturum recte pollicitum. D. 42, 8, 11 : Cassius actionem introduxit. M. Lenel, Pal. nos. 18 et 116, lui attribue le premier texte el le troisième, sans parler de sa préture. M.Krüger rapporte les deux premiers à sa préture qu'il admet avoir été la préture urbaine, p. 54, note 51. Il y a selon nous les mêmes raisons de décider pour lous, el, si on ne l'a pas aperçu pour le nôtre, c'est parce qu'on a voulu le mettre avec l'introduction soit de l'exceptio metus, soit de l'exceptio doli, soit d'autres moyens encore, dans une relation que ses termes contrarient en réalité beaucoup plus qu'ils ne l'imposent.

1) De off. 3, 14, 60 : protulerat de dolo malo formulas pourrait a l'extrème rigueur s'entendre de la formule de la cautio de dolo, comme on l'a soutenu jadis; mais de nat. deor. 3, 30, 74 : iudicium de dolo, quod C. Aquilius, familiaris noster protulit, ne peut se rapporter qu'à la création de l'action de dol. V. sur la conciliation de cette action de dol qui n'a pu être créée que par un préteur urbain ou pérégrin et de la quaestio ambitus présidée par Aquilius en 688, selon Cicéron, pro Cluentio, 53, 147 : C. Aquili, apud quem nunc de ambitu causa dicitur, Pernice, Labeo, 1, 58; Kipp, Krit. Vierteljahrschr. N. F. 12, p. 12 et ss. 2) V. les citations dans Pernice, Labeo, 2, 95, note 1. - ${ }^{3}$ ) V. la démonstration de Gradenwitz, Ungültigkeit, p. 17 et ss. 
qui serait tout au plus une transposition de l'original d'Apollodore de Karystios ${ }^{1}$ ). - - Quant aux interdits, il y en a des vestiges, à vrai dire singulièrement rares et menus: les passages du Stichus, représenté en 554, où l'on ne sait si le mot utrubi est une allusion ou une rencontre fortuite de la langue journalière et de la langue du droit ${ }^{2}$ ); l'allusion plus vraisemblable à l'exceptio vitiosae possessionis qui paralt se trouver dans l'Eunuque de Térence et qui attesterait l'existence de cette clause dans les interdits toujours avant 595 et plus précisément en 593 où la pièce fut probablement représentée ${ }^{3}$ ). Mais sans vouloir ici chercher dans quelle mesure les interdits ont pu primitivement être protégés en dehors de l'ordo iudiciorum par des multae ${ }^{4}$ ), nous remarquerons que rien n'indique à cette époque l'emploi de la formule arbitraire sine periculo qui seule attesterait l'introduction de la procédure formulaire. - Enfin nous terminerons en constatant que l'on ne rencontre pas dans les textes de la même période de témoignages plus décisifs de l'usage de la procédure formulaire dans la sphère des attributions des édiles curules $^{5}$ ). La juridiction édilicienne serait, croyons-nous, logi-

1) Phorm. 2, 4, 9 et $\mathrm{s}$ : quod te absente hic filius egit restitui in integrum aequomst et bonum et id inpetrabis. M. Bekker qui renvoie trois fois à ce texte dans son relevé instructif et complet des textes juridiques de Térence (Zeitschr. d. Sav.-Stift. 13, p. 53 et ss. II, 36. XVII, 23. XXIII, 2), ne s'est pas expliqué sur lui dans le passage de son commentaire oì il apprécie l'intrigue du Phormio, p. 111 et s. Mais il a précédemment réfuté, Aktionen, 2, 91, n. 32, l'argument qu'avait voulu en tirer Burchardi, Wiedereinsetanng, 154 et ss. 2) Stichus, 5, 4, 14 : nonc uter utvuli accumbanus. 5, 5, 9: utrubi accumbo. - utrubi tu vis. L'allusion à l'interdit est admise par Ubhelohde, dans Gilïck, Interd. 2, 337, 2 et par Karlowa, Rechlsgesch. 2, 1, 319. Elle parait donteuse à Costa, p. 457. - 3) Eunuch. 2, 3, 27: hance tu mihi vel vi vel clam vel precario fac tradas; mea nil refert dum potiar modo. Cf. Ubbelohde, loc. cit. Karlowa, p. 323. - ${ }^{4}$ ) V. les ohservations et les renvois de Pernice, Parergi, 2. Zeitschr. d. Sav.-Stift. 5, 33. -... •) Nous ne revenons pas ici sur les passages de Plaute dans lesquels on a cherché soit l'édit des édiles sur les vices: Capt. 4, 2, 43, soit les actions édiliciennes relatives à la vente : Merc. 2, 3, 86. Rud. 2, 3, 42. Mil. Glorios. 3, 1, 133. Ils nous paraissent toujours absolument incertains. V. Nouv. Rev. hist. 3, 415. En ce qui concerne particulièrement les doutes sur la force probante de Capt. 4, 2, 43, que M. Voigt critique, en les défigurant au reste un peu, Rechtsgesch. 1, 220, nous 
quement étrangère à notre débat, suivant la doctrine qui considère la procédure formulaire comme ayant été admise dans les rapports avec les pérégrins avant la loi Aebutia; mais, en réalité, on n'y trouve de traces avérées de cette procédure qu'à une époque où elle fonctionne également entre citoyens devant le préteur urbain. C'est même ce qui nous a dispensé de discuter ici la doctrine lont il s'agit.

En somme la littérature romaine explorée saus idée préconçue nous présente pendlant toute la durée du VI e siècle un dénuement absolu de formations juridiques impliquant l'existence de la procédure nouvelle. Rien n'y révèle autre chose que la procédure sobre et nue du système primitif où la legis actio que le magistrat n'a pas le droit d'accorder en dehors de la loi ne peut pas davantage être refusée par lui quand la loi la donne, où il n'y a pas de formules à côté d'elle, où par conséquent il n'est pas question non plus ni des exceptions, ni des actions prétoriennes, ni des actions de bonue foi qui n'ont pu naitre que dans le domaine des formules. Et ce dénuement se prolonge aussi radical, aussi absolu, sans qu'on ait même à lutter contre aucun des mirages auxquels il nous a fallu résistel précédemment, pendant les premières années du VIle siècle, jusqu'à la fin de son premier tiers à peu près. Au contraire depuis les environs de l'an 630 , il semble qu'on passe d'une couche géologique à une autre. Les formations incompatibles avec l'ancien système apparaissent de toute part dans les départements du droit. les plus variés. Nous nous bornons à les énumérer dans l'ordre chronologique.

1. Par un hasard qui s'accorde parfaitement avec la logique, le premier symptôme de l'ère nouvelle se rapporte à ce droit du magistrat d'accorder ou de refuser l'action qui n'a $\mathrm{pu}$, selon nous, être admis tant que la procédure des Actions de la loi domina sans partage, mais dont la reconnaissance a été la condition même de la juxtaposition de deux procédures. Il nous est attesté dès l'an 628 ou 629, par Cicéron, de orat.,

renverrons à Mommsen, Staatsrecht, $2,1^{3}, 508$, note 4 et Krüger, Rechtsquell. p. 32, note 8 . Les autres texles invoqués par M. Voigt pour établir l'existence de l'édit édilicien sur les actions redhibitoire et quanti minoris au milieu du VIe siècle appartiennent à une époque postérieure on sont étrangers à la questions. 
dans le récit du débat agité en présence de P. Mucius Scaevola, cos. 621, devant le préteur M. Crassus, entre Hypsaeus et Cn. Octavius, Hypsaeus demandant qu'on accorde à son client le droit d'intenter une action où il eut succombé pour plus pétition et $\mathrm{Cn}$. Octavius s'opposant à ce que son adversaire libère son client des risques et des ennuis de cette affaire de tutelle par l'exagération qui entrainerait l'échec de la demande').

Nous n'avons pas à nous occuper ici du point de savoir en quoi pouvait consister la plus petitio du client d'Hypsaeus ${ }^{2}$ ). Mais le texte implique évidemment que le magistrat a le droit de permettre ou de ne pas permettre au demandeur d'intenterpar legis actio, semble-t-il bien, - l'action qu'Hypsaeus lui demande d'admettre et Octavius d'écarter. Or le procès est au plus tôt de l'an 627, puisque Cn. Octavius, cos. en 626, est appelé consularis et il est all plus tard de 628 puisqu'Hypsaeus, cos. en 629 , ne reçoit pas la même qualification ${ }^{3}$ ). Il

1) De orat. 1, 36, 166: potes igitur, inquit Crassus, ... oratores putare eos, quos multas horas exspectarit, cum in campum properaret, et ridens et stomachans Scaevolr, cum Hypsaeus maxima roce, pluvimis verbis a M. Crasso praetore contenderet, ut ei, quem defendebat causa. cadere liceret, $C$ n. autem Octavius homo consularis non minus longa. oratione recusaret, ne adversarius causa caderet ac ne is, pro quo ipse diceret, turpi tutelae iudicio atque omni molestia stultitia adversarii liberaretur? 167 ... alter plus lege agendo petebat, quam quantum lex in $X I I$ tabulis permiserat; quod cum impetrasset, causa caderet: alter iniquum putabat plus secum agi, quam quod erat in actione, neque intelligebat, si ita esset actum, litem adversarium perditurum. - ${ }^{2}$ ) V. Lenel, Ed. p. 256, n. 10. Karlowa, Rechtsgesch. 2, 1, p. 281. - ${ }^{3}$ ) Le premier terme est signalé par Huschke, Multa, p. 496, n. 400, les deux par Wehrmann, Fasti praet. p. 12. L'attribution de la préture de Crassus à l'an 646 dans Drumann, 4, p. 70, note 11, est la reproduction d'une allégation gratuite de Pighius. C'est là le plus ancien témoignage daté sur la dénégation de la legis actio. Le texte de Val. Max, 7, 7, 3, qu'on cite si souvent comme la preuve de ce droit sans distinction d'époque, appartient sûrement à une période plus avancée de la procédure formulaire, où l'on connait déjà la $\boldsymbol{B} . \boldsymbol{P}$. unde cognati et où l'on est pour ainsi dire à la phase d'éclosion de la querella donné allx ascendants. Si le droit successoral qui y est supposé diffère cle celui exprimé dans les plaidoyers de Cicéron, c'est, croyons-nous, par un caractère plus développé. Sans pouvoir être à notre sens, rigoureusement démontrée, l'opinion selon laquelle l'egregia Gaii Calpurni Pisonis praetoris (Bern. et Paris: praefecti) urbis constitutio émạne de Gaius Calpumius Piso, 
atteste donc le droit du magistrat de denegare actionem en 627 ou 628.

2. Le témoignage que nous rencontrons ensuite est de l'an 631. C'est celui de la Rhétorique à Herennius qui représente deux préteurs, Sex. Iulius et M. Drusus, comme ayant diversement statué sur le transmissibilité des actions de mandat refusées contre les héritiers par le premier et données contre eux par le second ${ }^{1}$ ). La décision du premier, Sex. Iulius Caesar, qui fut préteur urbain en $631^{2}$ ), prouve l'existence à cette date de la loi Aebutia. C'est évident s'il s'agit de l'action de manlat réligée in factum dont les textes présentent des vestiges et avec laquelle s'accorderait très bien l'intransmissibilité. Mais c'est également vrai, s'il s'agit de la formule in ius, puisque cette formule in ius, concurrente plus jeune de la formule in factum, n'a pu naître qu'après elle ${ }^{3}$ ).

3. Les actions prétoriennes données à la suite de la bonorun venditio dont l'intentio était au nom du failli et la condemnatio à celui de l'acheteur des biens, ont été inventées,

cos. 687 el préteur au plus tard en 684 (Drumann, 2, 92, n. 25; Wehrmann, p. 45; Leist, dans Glück, Serie d. Büch. 37, 38, 1, p. 74 et s. Voigt, Rechtsgesch. 1, p. 540, n. 38), nous parait avoir pour elle tontes les vraisemblances.

1) Ad Her. 2, 13, 19: M. Drusus, praetor urbanus, quod cum herede mandati ageretur, indicium reddidit, Sex. Iulius non reddidit. -- 2) La date de la préture de Sex. Iulius est fonmie par un texte de Cicéron, de domo, 53, 136: cum Licinia virgo Vestalis ... T. Flaminio Q. Metello consulibus (an d. R. 631) aram et aediculam et pulvinar sub saxo dedicasset, nonne eam rem ex auctoritate senatus ad hoc collegium Sex. Iulius praetor rettulit? Quant au caractère urbain de cette préture, il est déjà rendu vraisemblable par le même texte: c'est principalement. le préteur urhain qui a de tels rapports avec le sénat en l'absence des consuls. Mais il résulte dı texte précédent: l'auteur ne peut comparer les denx préteur's que comme étant les deux préteurs urbain et pérégrin de la mème année on comme étant deux préteurs de la même catégorie de deux années différentes; or Sex. Inlius n'a pas été préteur la même année que $M$. Livius Drusus dont la prétıre mrlaine se place, verronsnous, au plus tôt en l'an 634; il doit done avoil' été préteur urbain comme lui, - ${ }^{3}$ ) Nous n'osons faire figurer jei l'action en restitution de dot intentée vers 633 devant $P$. Mucius Scaevola par la veuve de C. Gracchus (D. 25, 3, 66); car sans parler d'autres difficultés soulevées par l'action rei uxoriae, nous ne croyons pas certain, que comme le pense M. Karlowa, Rechtsgesch. 2, 1, p. 215, il s'agisse là de l'action rei 
selon Gaius, 4, $35^{1}$ ), par le préteur P. Rutilius que l'on disait avoir été le créateur de la bonorum venditio. Quel est ce P. Rutilius? C'est forcément le seul jurisconsulte portant ce nom et ce prénom, le seul personnage qu'on puisse vouloir désigner dans un ouvrage de droit quand on emploie ce nom et à plus forte raison ce prénom, le consul de 649 P. Rutilius Rufus, dont une argumentation probante permet de placer la préture en 636 au plus tard. On a parfois prétendu y voir un P. Rutilius plus ancien par exemple le préteur de 548, P. Rutilius Calvus, cité par Tite-Live, 45, 44, 2, et il a pu y avoir encore d'autres préteurs inconnus appelés P. Rutilius, puisque nous n'avons pas la liste complète des préteurs, ni spécialement des préteurs chargés de la justice civile. Mais en réalité l'assignation ne comporte pas de doute. Quand un jurisconsulte romain parle de P. Rutilius ou de Rutilius tout court, il ne peut pas y avoir plus d'hésitation que quand un jurisconsulte moderne parle de Savigny ou de Pothier, de Thibaut ou de Dupin. Le renvoi à la préture de Rutilius est aussi clair que le serait un renvoi à une mémoire de Savigny ou à des conclusions de Dupin. Il s'agit du jurisconsulte; car, s'il s'agissait d'un homonyme, on spécifierait afin d'empêcher la confusion ${ }^{2}$ ). Quant à la date de la préture de P. Rutilius Rufus, elle est fixée, au moins d'un côté, par le fait que Rutilius brigua le consulat contre M. Aemilius Scaurus et, après avoir été battu, entama avec lui une seconde lutte de poursuites réciproques dans laquelle il succomba de nouveau ${ }^{3}$ ). L'histoire ne connaît qu'un consulat de M. Aemilius Scaurus : celui qu'il occupa en 639 avec M. Caecilius Metellus. C'est donc pour cette année que Rutilius brigua le consulat sans succès et, en vertu des règles sur la succesion et l'intervalle des magistratures,

uxoriae plutôt que d'une action ex stipulatu née d'une cautio rei uxoriae.

1) Gaius, 4, 35: quae species actionis appellatur Rutiliana, quia a praetore $P$. Rutilio, qui et bonorum venditionem introduxisse dicitur, comparata est. $-{ }^{2}$ ) Huschke, Zeitschr. de Linde, 14, p. 19, n. 4, relève avec raison la façon dont est précisément pour cela spécifié le cognomen du Rutilius Maximus auteur du fr. 125, D. 30. $-{ }^{3}$ ) Cicéron, Brut. 30, 113: cum una consulatum petiissent, non ille (P. Rutilius) solum, qui repulsam tulerat, accusavit ambitus designatum competitorem, sed Scaurus etiam absolutus Rutilium in iudicium rocavit. Cf. de or. 2, 69, 280.

Zeitschrift für Rechtsgeschichte. XIV. Rom. Abth. 
il doit donc avoir été préteur au plus tard deux ans auparavant en $636^{1}$ ). Il est vrai qu'on a parfois voulu placer le procès en une année différente ell admettant que Scaurus aurait occupé une seconde fois le consulat, comme consul suffect, en 647 après la mort de L. Cassius Longinus ${ }^{2}$ ). Mais cette allégation qui permettrait de faire descendre la préture de Rutilius jusqu'en 644 au plus tard, ne s'appuie sur aucun texte $^{3}$ ) et elle suppose une infraction à la défense dont est frappée l'itération du consulat de 603 environ à $673^{4}$ ). La date de 636 paraît d'ailleurs la plus généralement admise. Seulement on remarquera que c'est exclusivement une date extrême au dessous de laquelle la préture de Rutilius ne peut descendre et non pas, comme on a souvent l'air de croire, la date précise de cette préture; car, sans parler d'autres difficultés accessoires, nous ne savons pas en quelle année est né Rutilius ${ }^{5}$ ), ni par conséquent à partir de quand il a été

1) Huschke, loc. cit. p. 4, invoque un autre argument : les individus condamnés pour brigue auraient été dès alors inéligibles pour dix ans, ce qui ferait remonter la condamnation de Rutilius, consul en 649, à 638 . Mais, quoique certains auteurs (v. par ex. Herzog, Gesch. u. Syst. d. römisch. Staatsverfass. 1, 658, n. 5) fassent encore remonter la loi Cornelia qui établit cette peine d'après schol. Bob. in Cic. pro Sull. 5, 17, aux consuls de 573 de Tite-Live, 40, 19, nous la croyons plutôt de L. Cornelius Sulla. V. Mommsen, Staatsrecht, $1^{3}$, 492, 1 . $3=$ tr. f. 2, 141, n. 2. - ${ }^{2}$ ) V. en ce sens Drumann, 1, 27. Pauly, Realencycl. 1, 37. 6, 587. Orelli, onomastjc. v. M. Aemilius Scaurus. M. Voigt, qui invoque ces auteurs, Rechtsgesch. 1, p. 217, n. 4, et qui proclame même la date de 636 » interdite par les faits historiques concomitants *, commet en tout cas certainement une erreur en plaçant le procès en 646 ; car, si Scaurus avait été candidat pour 647 , ce n'aurait pu être qu'après la mort de Cassius, en 647. Pour mettre le procès en 646, il faudrait confondre M. Aemilius Scaurus, avec M. Aurelius Scaurus, cos. suff. en 646, suivant une erreur autrefois répandue et contre laquelle un des auteurs consultés par M. Voigt, Drumann met déjà en garde. $-{ }^{3}$ ) Elle est même contredite par le texte de Cicéron, Brut. 30, 113, qui suppose le procès fait entre la désignation et l'entrée en charge de Scaurus; car, si Scaurus avait été élu à la place rendue vacante par la mort de Cassius, on n'eut pas laissé entre l'élection et l'entrée en fonctions le temps de faire des procès. - 4) Tite-Live, ep. 56. Mommsen, Staatsrecht, $1^{3}, 521$, n. $1=$ tr. f. 2,174, n. $\left.1 .-5\right)$ La date de 596, proposée par Maiansius et depuis souvent reproduite (par ex. dans Tenffel-Schwahe, $1^{5}$, p. 232) est fondée par lui uniquement sur l'idée qu'il faudrait dès alors l'âge de 43 ans pour être éligible au 
éligible à la préture. Ce que l'on peut, croyons-nous, dire de plus précis, c'est qu'ayant été tribun militaire en $620^{1}$ ) et ayant par suite déjà fait alor's les cinq stipendia dont le cours ne commençait qu'à dix sept ans accomplis ${ }^{2}$ ), il est né au plus tard en 598 et que, si l'on réfléchit qu'il vivait encore en l'an 676 où Cicéron le visita à Smyrne et probablement en 677 où le de natura deorum l'y suppose toujours ${ }^{3}$ ), il faudrait lui attribuer une grande longévité pour le supposer né beaucoup auparavant. Cela permettrait, en partant des âges admis par M. Mommsen pour la période antérieure à Sulla ${ }^{4}$ ), de placer sa préture au plus tôt en 628 , s'il n'a pas été édile curule, ou en 631 , s'il a été édile curule, comme un indice porte à le croire ${ }^{5}$ ). Mais ce sont là des approximations qui perdent de leur valeur à mesure qu'elles se compliquent. Ie principal pour notre sujet est que la préture de Rutilius vienne attester l'existence de la loi Aebutia au plus tard en l'an 636 .

4. C'est sans doute à la préture de 636 au plus tard du même P. Rutilius Rufus qu'il faut rapporter l'édit restreignant les droits dı patron à l'égard de l'affranchi qui est cité par Ulpien comme émanant du préteur Rutilius $\left.{ }^{6}\right)$.

5. Nous trouvons ensuite le préteur urbain M. Livius Drusus qui donnait l'action de mandat contre les héritiers. Sa préture ne peut se placer après 639 , puisqu'il fut consul

consulat. Elle ne donnerait donc elle-même qu'un terme extrême. Il n'y a, en présence de l'incertitude de la terminologie romaine rien à tirer des textes de Cicéron, qui représentent Rutilius comme adulescentulus en 616 (Brut. 22, 85) et adulescens en 625 (ad Att. 4, 15, 2).

1) Appien Hisp. 88. Cicéron, de re p. 1, 11, 17. - 2) Mommsen, Staatsrecht, $1^{3}, 505$, n. 2.506, n. $2=$ tr. f. 2,156, n. 2.157, n. 2. Cf. 23,576, n. 4. - ${ }^{3}$ ) Brut. 22, 85; de re p. 1, 8, 13; de nat. deor. 3, 32, 80. - ${ }^{4}$ Staatsrecht, $1^{3}, 565$ ss. $=$ tr. fr. 2,225 ss. Les termes seraient forcément plus resserrés dans la doctrine qui exige dès alors l'âge de 40 ans et qui contraint même, avons-nous vu, à rejeter la naissance de Rutilius à 596 au plus tard. - s) Suélone, Aug. 89: orationem ... Rutili de modo aedificiorum. - $\left.{ }^{\circ}\right)$ D. 38, 2, 1, 1: et quidem primus praetor Rutilius edixit se amplius non daturum patrono quam operarum et societatis actionem, videlicet si hoc pepigisset, ut nisi ei obsequium praestaret libertus, in societatem admitteretur patronus. Cf. Mommsen, Staatsrecht, 3, 433, n. $1=$ tr. f. 6, 2, 16, n. 4 . 
en $642^{1}$ ). Mais un instrument qui a jusqu'à présent été négligé permet de la localiser dans l'autre sens. M. Drusus a été tribun du peuple en 632 , lors du second tribunat de C. Gracchus ${ }^{2}$ ). Il ne peut donc avoir été préteur avant 633 , ni, si l'on se rappelle l'intervalle d'un an exigé par la loi Villia après l'occupation du tribunat ${ }^{3}$ ), avant 634 , ni même probablement, en tenant compte du délai de quatre ans qui n'était pas requis légalement mais qui était tout à fait usuel entre le tribunat et la préture ${ }^{4}$ ), avant 637 . La date de sa préture se place donc certainement entre 634 et 639 , probablement entre 637 et 639 .

6. C'est y a-t-il tout lieu de penser, au même Drusus et par suite à la même date qu'il faut rapporter l'édit du préteur Drusus invoqué par Cicéron comme refusant la vindicatio in libertatem à l'affranchi qui ne voulait pas répéter le iusiurandum liberti après l'affranchissement ${ }^{5}$ ).

7. En 643, nous rencontrons le premier monument épigraphique qui porte des traces positives de la procédure nouvelle. C'est la loi agraire, qu'on a longtemps prise pour la loi Thoria, que l'opinion dominante admet aujourd'hui ne pas être elle, mais que tout le monde reconnait dater de l'an $\left.643^{6}\right)$. Il faut d'abord y relever la disposition autorisant à faire la professio qu'elle prescrit, à la place du débiteur saisi : 1) l'acquéreur qui tient du magister bonorum, du curator, du bonorum emptor, puis 2) sans doute auparavant le magister, le curator et l'emptor eux-mêmes parmi lesquels le curateur seul est resté nommé dans les débris de la loi immédiatement précédents ${ }^{7}$ ). C'est, nettement visé, tout le personnel de la venditio

1) Wehrmann, Fasti praet. p. 15. - ${ }^{2}$ ) Gicéron, Brut. 28, 109 : M. Drusus $C$. $f$. qui in tribunatu C. Gracchum collegam iterum tribunum fregit. $-{ }^{3}$ ) Mommsen, Stantsrecht, $1^{3}, 534$, n. $3=$ tr. f. 2, 189, n. 3. 4) Cicéron all Att. 12, 5, 3 et l'interprétation de Mommsen, Staatsrecht, 1 , 552, n. $3=$ tr. f. 2,211 , n. 1. Gf. Nipperdey, leges annales, p. 34, 35.${ }^{5}$ ) ad Att. 7, 2, 8: itaque usurpavi vetus illud Drusi, ut fer'unt, praetoris in eo, qui eadem liber non iuraret, me istos liberos non addixisse, praesertim cum adesset nemo, a quo recte vindicarentur. - $\left.{ }^{\circ}\right) \mathrm{V}$. les développements donnés $C$. I. I. I, 200. Cf. Karlowa, Rechtsgesch. 1, p. $433-437 .-{ }^{7}$ ) Ligne $56:$ [u]tei curator eius profiteatur, item ute[i ... ex e]o edicto, utei is, quei ab bonorum emptore magistro curato[reve emerit] et les observations de Mommsen. 
bonorum, aussi nécessairement postérieur à la loi Aebutia que la venditio et les moyens prétoriens qui la sanctionnent.

On peut encore, à notre sens, voir un autre vestige de la procédure nouvelle dans les exceptions ou peut-être mieux les prescriptions qui sont énumérées dans la loi comme opposables aux poursuites des publicains ${ }^{1}$ ) et qui ont été récemment mises à contribution pour l'histoire de l'effet extinctif de la litis contestatio ${ }^{2}$ ), de même qu'elles pourraient d'ailleurs l'être pour celle du paiement ${ }^{3}$ ). Il n'y a pas là une preuve directe; car il ne s'agit pas dans ce passage de juridiction civile de la compétence exclusive des deux préteurs urbain et pérégrin. Il s'agit de juridiction administrative de la compétence des censeurs et à leur défaut des consuls et des préteurs ${ }^{4}$ ). Tout ce dont il peut être question, c'est d'une imitation des formes de la procédure civile ${ }^{5}$ ). Mais, quand on se trouve en face de particularités de procédure aussi techniques et aussi délicates que celles relatives aux moyens tirés de la chose jugée, de la déduction en justice ou de la litispendance, on ne peut guère songer à un développement spontané du droit public, il n'y a pas de témérité à croire à une imitation du droit privé. On peut admettrc sans scrupules la copie d'un modèle préexistant.

Nous ferions infiniment plus de réserves sur un troisième ordre de vestiges auquel on pourrait songer. La même loi donne, avant de mentionner ces défenses, une action aux publicains pour défaut de paiement du vectigal ${ }^{6}$ ). Il pourrait

1) Ligne 38: [de ea re iudicare iubeto,] quae res soluta n[on siet inve iudici7o non siet iudicatave non siet, quod eius praevaricationus [causa ... vel per dolum malum petitorum patronoru]mve factum non siet. - 2) Eisele, Abhandl. zum röm. Civ.-Proc. p. 19 et ss. - ${ }^{3}$ ) Les explications opposées par Eisele, p. 21, à l'argument qu'on pourrait tirer des mots quae res soluta non siet ne nous paraissent point absolument décisives. - 4) Voir le commencement du texte plus bas note 6 . $\left.{ }^{5}\right)$ M. P. Krüger, Krit. Vierteljahrschr. N. F. 13, 323, discute la force probante du texte à un autre point de vue qui nous semble moins exact. - ') Ligne 36 et ss. : Quoi publicano e(x) h. l. pequunia debebitu[r ... Sei quid publicanus eius rei causa sibi deberi] darive oportere de[icat, de ea re cos. prove con]s(ule) pr(aetor) prove pr(aetore), quo in ious adierint ... [recuperatores ex ci[vibus $L$, quei classis primae sient, XI dato, ... [quod ita ioudicatum eJrit ... utei is quei indicatus erit dare oport[ere solvat]. 
sembler naturel de voir là l'action fictice mise, selon Gaius, à la place de la piguoris capio des Actions de la loi ${ }^{1}$ ). Mais cette idée, qui a été exprimée ${ }^{2}$ ), soulève des objections graves. Sans parler de ce que pas une allusion n'est faite à l'existence d'une fiction, le domaine de l'action ne concorde guère avec celui de la pignoris capio ${ }^{3}$ ). Enfin et surtout, s'il s'agissait de l'action fictice de la pignoris capio, elle serait, comme la pignoris capio elle-même, de la compétence exclusive des tribunaux civils. Or, ainsi que nous venons de remarquer, elle n'est pas déférée exclusivement aux préteurs : elle l'est avant eux aux consuls et, si elle ne l'est pas en première ligne aux censeurs, c'est qu'il n'y en avait pas alors en fonctions. Il ne s'agit donc là que d'un procès administratif dont on peut d'autant moins argumenter pour la procédure ordinaire qu'il s'agit d'un rapport plus simple.

8. Soit peut-être en partie dès avant 643 , soit ensuite dans une période qui s'étend jusqu'en l'an 672 , on rencontre dans la carrière administrative et scientifique de $Q$. Mucius Scaevola le pontife, gouverneur d'Asie vers 656, consul en 659, mort en 672, un ensemble particulièrement varié de documents relatifs à la procédure formulaire. Nous ne les dépouillerons pas tous. En laissant de côté les solutions de détail éparses dans le Digeste, pour lesquelles, sans parler de la question d'exactitude de transmission, la discussion nous entraînerait dans des débats compliqués de droit privé, en laissant aussi de côté l'exception accordée dans l'édit d'Asie ${ }^{4}$ ), qu'on pourrait à la rigueur vouloir écarter comme se référant à des procès pérégrins, nous en citerons seulement deux: le texte connu de Cicéron, selon lequel Q. Mucius a exercé une influence sur l'élaboration du système déjà constitué des actions de bonne foi ${ }^{5}$ ); le texte du Digeste selon lequel il a

1) Gaius, 4, 32: in ea forma quae publicano proponitur, talis fictio est, ut quanta pecunia olim, si pignus captum esset, id pignus is a quo captum erat luere deberet, tantam pecuniam condemnetur. - ${ }^{2}$ ) V. Karlowa, Leyisactionen, p. 216. - 3) Pernice, Parerga, 2. Zeitschr. d. Sav.-Stift. 5, pp. 66, 67. Cf. Wlassak, Processgesetze, 1, 90. 91. 252, n. 26. - 4) V. p. 26, note 1. - 5) De off. 3, 17, 70: Q. quidem Scaevola pontifex maximus summam vim esse dicebat in omnibus iis arbitriis, in quibus adderetur »ex fide bona \& fideique bonae nomen existimabat manare latissime idque versari in tutelis societatibus fiduciis mandatis 
commenté l'édit sur l'homme libre qui s'est laissé vendre pour partager le prix ${ }^{1}$ ).

Après Q. Mucius Scaevola, les témoignages deviennent si abondants et si connus qu'il serait superflu de les rassembler. $\mathrm{Ce}$ que nous avons dit suffit pour justifier pleinement la détermination de notre premier terme, pour établir que la loi Aebutia existe sârement avant la mort de ce jurisconsulte survenue en 672, avant le vote de la loi agraire de 643 , avant la préture de Drusus, au plus tard en 639, avant celle de P. Rutilius Rufus, au plus tard en 636, avant celle de Sex. Iulius, au plus tard en 631, avant celle de P. Crassus, au plus tard en 628, tandis qu'on n'en trouve auparavant aucun vestige avéré. Et nous remarquerons que, si, comme la vérité scientifique nous paraissait l'exiger, nous avons fait rentrer dans notre argumentation les textes relatifs aux actions de bonne foi et au droit du magistrat de refuser l'action, elle peut, dans ses termes essentiels, être maintenue en dehors d'eux. Naturellement la preuve négative faite pour le VIe siècle et le début du $\mathrm{VII}^{\mathrm{\theta}}$ quant au cercle large l'est du même coup quant au cercle étroit. Mais la preuve positive, qui se réfère à la période suivante, peut également subsister, à deux ou trois ans près, en éliminant les termes controversés; car il reste alors, pour Q. Mucius, l'édit sur l'homme libre qui s'est laissé vendre, pour la loi agraire, les prescriptions et la bonorum venditio, pour M. Livius Drusus l'édit relatif au iusiurandum liberti, pour Rutilius l'édit sur les operae et

rebus emtis venditis conductis locatis quibus vitae societas contineretur; in iis magni esse iudicis statuere, praesertim cum in plerisque essent iudicia contraria, quid quemque cuique praestare oporteret. Sur l'interprétation du texte, v. en dernier lieu H. Krüger, Zeitschr. d. Sav.-Stift. 11, 195 et ss.

1) Paul, D. 40, 12, 23 pr. Le ms. de Florence porte Quintus meus au lieu de Quintus Mucius et cette leçon a été défendue par M. Kipp, Zeitschr. d. Sav.-Stift. 9. p. 164, qui la rapporte à Quintus Cervidius Scaevola. Mais M. Kipp n'a pu établir que Paul, qui appelle Q. Cervidius Scaevola Scaevola noster ou tout au plus Quintus Cervidius Scaevola noster (cette formule isolée de D. 28, 6, 38, 3, n'est probablement qu'une glose, a justement remarqué P. Krüger), l'ait jamais appelé Quintus meus en disant Quintus tout court au lieu de Scaevola et meus au lieu de noster. 
les formules rutiliennes et même probablement pour Sex. Iulius l'action mandati conçue in factum ${ }^{1}$ ).

\section{II.}

Maintenant jusqu'à quand la procédure des Actions de la loi est-elle demeurée seule? Comment fixer l'autre terme extrême, le moment où la loi Aebutia ne peut pas encore exister? Il faut ici de nouveau procéder par ordre.

1. La loi Aebutia ne peut d'abord pas, suivant une doctrine très répandue et que nous croyons vraie ${ }^{2}$ ), être antérieure à la publication par Sex. Aelius Paetus Catus de ses tripertita, qui, selon la relation connue de Pomponius ${ }^{3}$ ), commençaient par les XII tables, continuaient par l'interpretatio et finissaient par la legis actio. On peut discuter sur le caractère et le plan de l'ouvrage de Sex. Aelius. On peut se demander si, conformément à l'opinion la plus accréditée, il s'agit là d'une division générale du livre ou si, conformément à une conjecture de Huschke ingénieusement reprise par M. Lenel ${ }^{4}$ ), ce ne serait pas pour chaque matière que Sex. Aelius donnerait successivement le texte des XII tables, l'interprétation et la legis actio. On peut encore chercher quel était, soit dans un plan, soit dans l'autre, le rapport de la première section et de la seconde, si le ius Aelianum mentionné ailleurs par Pomponius se confond avec l'ouvrage ou avec sa troisième partie $^{5}$ ). Il reste, en tout cas, malaisé d'admettre que la

1) Cette argumentation ne cesserait d'ètre probante que pour les auteurs, qui, comme MM. Jörs ct Wlassak, admettent qu'il y a eu avant la loi Aebulia des édits prétoriens, des actions prétoriennes, et sans doute aussi, logiquement, des exceptions prétoriennes. Mais alors, à vrai dire, il est impossible de prouver l'existence de la loi Aebutia à une époque quelconque antérieure aux textes d'Aulu-Gelle et de Gaius, ou tout au moins aux lois Inliae. - ${ }^{2}$ ) C'est la doctrine admise par presque tous les auleurs cités p. 11, note 2, qui adoptent une date postérieure au milieu du VI e siècle. - $\left.{ }^{3}\right)$ D. 1, 2, 2, 38: . . exstat illius liber qui inscribitur tripertita, qui liber veluti cunabula iuris continet: tripertita autem dicitur, quoniam lege duodecim tabularum praeposita iungitur interpretatio, deinde subtexitur legis actio. - ${ }^{4}$ ) Huschke, Zeitschr. I. gesch. Rechtswiss. 15, p. 179 ss. Lenel, Sabinussystem, p. 10. - 5) Cf. outre Lenel, op. cit. p. 9, P. Krüger, Gesch. d. Quell. p. 54, п. 16. Karlowa, Röm. Rechtsgesch. 1, 476. A. Pernice, dans Holtzendorff, p. 134 et Zeitschr. d. Sav.-St. 7, 2, 160. 
section dernière relative à la procédure eut pu être désignée comme relative aux legis actiones, s'il eut existé à côté d'elles une autre procédure que l'auteur eut nécessairement expliquée là. Or, Sex. Aelius a été édile curule en 554, il a été consul en 556, puis censeur en $560^{1}$ ). Cela donne comme date approximative où la procédure formulaire n'existe pas encore le milieu du VI ${ }^{e}$ siècle. Mais on peut descendre plus bas.

2. La loi Aebutia ne peut, d'après l'ordre suivi par Gaius dans son exposé du système des Actions de la loi, ni surtout d'après les vraisemblances générales, être antérieure à la loi Vallia qui transforma la manus iniectio en manus iniectio pura dans tous les cas sauf deux ${ }^{2}$ ). Or la loi Vallia appartient au plus tôt à la seconde moitié du VI e siècle. En effet, d'après le langage de Gaius, elle est plus récente que la loi Furia legatorum ${ }^{3}$ ). Et, d'autre part, M. Bruns ${ }^{4}$ ) a établi par

1) Tite-Live, 31, 50. Fastes du Capitole. - 2) Gaius, 4, 25. 3) Gaius, 4, 23 : sed aliae leges ... constituerunt quasdam actiones ... veluti lex Furia testamentaria ... 25 : sed postea lege Vallia ... 4) Bruns, Zeitschr. f. Rechtsgesch. 12, 127 et ss. = Kl. Schr., 2, 305 et ss. Bruns a invoqué un autre argunent qui prouverait d'ailleurs seulement que la loi Vallia n'existait pas dans la première moitié du VI ${ }^{\text {e }}$ siècle. Il est liré de l'inscription de Luceria, C. I. L. IX, 782, qui, d'après les caractères et la laugue, appartient à cette époque et qui, en matière de voirie, établit une action populaire sous forme de manus iniectio pro iudicato. C'est, a pensé Bruns, la preuve que la loi Vallia restrictive de la manus iniectio pro iudicato n'était pas encore rendue. Mais, comme l'a par exemple justement remarqué M. Wlassak, Processgesetze 1, 192, n. 34, Luceria est une colonie latine (Cf. C. I. L. IX, p. 74 ss.) qui vit théorjquement selon son droit local, si les lois romaines peuvent en fail s'y retrouver plus ou moins largement en vertu d'emprunts volontaires ou forcés (v. à ce sujet en termes un peu divergents Th. Mommsen, Röm. Staatsrecht, 3, 692. 695. $700=$ tr. fr. 6, 2, 323. 326. 332, et Wlassak, Processgesetze, 2, 152 ss. 179, n. 29). La disposition relative à la manus iniectio pro iudicato peut y ètre un emprunt au droit romain. La loi Vallia peut aussi, soit par la volonté des conices de Rome, soit par celle de l'assemblée de Luceria, être passée du droit romajn dans le droit local de cette cité. Mais il ne nous est prouvé ni que cette loi ait été immédiatement imposée aux Latins, ni qu'elle ait été plus ou moins rapidement adoptée par le peuple de Luceria. On pourrait aussi bien admettre que, soit à Luceria soit ailleurs, le droit ancien de la manus iniectio ait, comme d'autres iustitutions archaïques conservées par tel ou tel groupe latin, subsisté dans sa rigueur première chez les Latins d'Italie jusqu'au jour où 
un raisonnement ingénieux que la loi Furia, qui a précédé la loi Voconia de $585^{1}$ ) n'est elle-même venue qu'après la loi Cincia de 550, puisqu'elle introduit en matière de dispenses fondées sur la parenté un système nouveau différent de celui que suit encore la loi Cincia ${ }^{2}$ ). La loi Furia se place donc entre 550 et 585 , dans la seconde moitié du VI ${ }^{e}$ siècle: ce qui rejette un peu plus bas, dans la seconde moitié du VI ${ }^{\circ}$ siècle, la loi Vallia. Et la loi Aebutia, postérieure elle-même à la loi Vallia, est forcément venue encore un peu plus tard. Même en resserrant beaucoup les trois lois, cela ne peut mener bien loin de la fin du VI ${ }^{e}$ siècle.

3. Un terme plus récent et plus précis est fourni, à mon sens, par deux monuments rigoureusement datés qui impliquent l'existence exclusive de la procédure des Actions de la loi en l'an 605 de Rome et même un peu après. Je parle des deux lois Calpurnia et Iunia de repetundis signalées par la loi Acilia de 631 ou 632 comme ayant permis avant elle d'intenter les poursuites de repetundae par voie de sacramentum ${ }^{3}$ ).

La loi Calpurnia est, suivant un texte positif de Cicéron ${ }^{4}$ ), de l'an 605. La loi Iunia est certainement postérieure sans qu'on puisse déterminer plus nettement la date où elle se place entre l'an 605 et l'an $632^{5}$ ).

ils devinrent citoyens. Nous ne savons; mais, précisement pour cela, l'existence de la manus iniectio pro iudicato à Luceria n'empèche pas celle de la loi Vallia à Rome.

1) Gaius, 3, 225: itaque lata est lex Furia ... 226: ideo postea lata est lex Voconia. Voir, quant aux dates des lois Cincia et Voconia la p. 25, note 2 et Cicéron, De senect. 5. - ${ }^{2}$ ) Fr. Vat. 298. 299. 301 et les explications de Bruns. $-{ }^{3}$ ) C. I. L. 1, 198; Bruns, Fontes s, p. 63 et ss., ligne 23: [quci pecuniae captae condernnatus est erit aut quod cum eo lege Calpu]rnia aut lege Iunia sacramento actum siet aut quod h. l. nomen [delatum sie]t. Cf. lignes 74-81. - 4) Cicéron, Brut. 27, 106: quaestiones perpetuae hoc (C. Papirio Carbone cos. a.634) adulescente constilutae sunt, quae antea nullae fuerunt: L. enim Piso tr. pl. legem primus de pecuniis repetundis Censorino et Manilio cos. (a. 605) tulit. - ${ }^{5}$ ) Elle ne peut être du tribun de 628, M. Iunius Pennus, comme parait le croire encore M. Karlowa, qui la dit, Röm. Rechtsgesch. 1, 432, probablement de l'an 628; car le père de M. Iunius Pennus portait le prénom de Marcus (Cicéron, Brut. 28, 109) et la loi Acilia nous apprend, ligne 74, que l'auteur de la loi Iunia s'appelait M. Iunjus D. f. Suivant une conjecture de Borghesi, opp. 5̃, 169, qui est vraisemblable, 
Or il est aujourd'hui de plus en plus reconnu ${ }^{1}$ ), malgré le laconisme de nos informations et l'abandon duquel souftient les études de droit criminel romain, que nos deux lois, à la première desquelles on rattache l'origine du système des quaestiones perpetuae, n'ont aucunement eu pour caractère d'organiser des tribunaux répressifs mis à la place des comices judiciaires et chargés de prononcer des peines publiques contre des crimes; qu'elles ont simplement permis contre des magistrats concussionnaires au profit de particuliers lésés des poursuites privées. Le développement hybride selon lequel la procédure des quaestiones a, sans jamais perdre complètement la marque de son origine civile, fini par envahir, dans le cours du VII ${ }^{\theta}$ siècle, tout le domaine du droit criminel est le terme de l'évolution; il ne doit pas égarer sur son début. Au début, marqué précisément par nos lois, il n'y a trace ni de peine publique, ni de procédure criminelle, ni de tribunal répressif. L'objet du procès n'est pas de faire prononcer la mort ou une amende au profit de l'Etat; il est de faire rendre aux sujets qui l'intentent ce qui leur a été enlevé par des magistrats malhonnêtes, sans même que, comme dans les actions pénales, elles aussi rigoureusement privées, qui naissent des délits, la restitution porte sur un multiple; car le montant de l'action qui, à partir de la loi Acilia, fut du double comme dans l'action furti nec manifesti et qui devint plus tard du quadruple comme dans l'action furti manifesti, est encore ici du simple comme dans la condictio furtiva ${ }^{2}$ ). L'autorité compétente

mais qui n'est que vraisemblable, il faudrait l'attribuer au consul de l'an 645, M. Iunius Silanus, qui, en sa qualité d'ıomme nouveau peut avoir parcouru lentement la carrière des honneurs et avoir élé tribun, dès l'an 620, par exemple, suppose Mommsen, C. I. L. I, p. 55.

2) Monımsen, C. I. L. I, p. 65. Rörn. Staatsrecht, $1^{3}, 182.2^{3}$, 223 s. $=$ tr. f. $1^{2}, 192.3$, 256 ss. V. dans le même sens Keller, Röm. Civilprocess, § 16, note 226; Maynz, N. R. hist. de droit, 6, p. 2, n. 5; Jörs, Röm. Rechtswissenschaft, 1, p. 188, 11. 1; Schulin, Lehrbuch d. Gesch. d. rörn. Rechts, pp. 540. 541; M. Voigt, Rörn. Rechtsgesch. 1, pp. 710. 711; A. Pernice, Labeo, 3, 1, p. 233, et plus anciennement les développements de C. Th. Zumpt, De legibus iudiciisque repetundarum, Berlin, 1845, p. 9 et ss. - ${ }^{2}$ ) C'est ce qui résulte de l'opposition faite entre les deux régimes par la ligne 59: [quod ante h.l. rogatam consilio probabitur captum coactum abjlatum avorsum conciliatumve esse, eas 
n'est pas celle qui exerce la juridiction criminelle; c'est celle à laquelle sont déférés de droit commun et sont même seuls déférés à cette époque les procès civils: le préteur, et puisqu'il s'agit de procès intentés par des pérégrins, le préteur pérégrin ${ }^{1}$ ). Enfin le procès ne s'intente pas davantage selon la forme observée depuis des siècles pour les procès criminels, dans la forme d'une cognitio du magistrat suivie le cas échéant d'une provocatio ad populum. Il s'intente dans la forme que Gaius indique comme étant, au temps des Actions de la loi, celle de tous les procès civils pour lesquels une loi spéciale n'avait pas établi une procédure différente ${ }^{2}$ ), dans la forme de la legis actio sacramenti ${ }^{3}$ ). Il n'est même pas

res omnis simpli, ceteras res omnis, quod post hance legem rogatam co[nsilio probabit]ur captum coactum ablatum avorsum conciliatumve esse, dupli. V. Mommsen, C. I. L. I, p. 65. 70. Zumpt, p. 11.

1) C'est encore lui, comme on sait, à qui la ligne 16 de la loi Acilia laisse la présidence de la quaestio pour l'année courante, tandis qu'elle a plus tard à sa tète un préteur spécial, probablement en vertu de cette loi, Mommsen, C. I. L. I, p. 65 et Staatsrecht, 2, 200, n. $1=$ tr. fi. 3, p. 229, n. 1. Zumpt p. 11. - 2) Gaius, 4, 13. - ${ }^{3}$ ) C'est l'opinion d'à peu près tous les auteurs cités p. 43 , note 1. M. Pernice entend le texte d'une façon également admissible grammaticalement, selon laquelle la loi Acilia n'attesterait l'emploi du sacramentum qu'en vertu de la loi Iunia. Mais cela ne l'empêche pas de penser que la loi Calpurnia ait établi une procédure civile; car précisément il propose cette interprétation à l'appui d'une conjecture hardie par laquelle il identifie la loi Calpurnia de repetundis avec la loi Calpurnia qui, selon Gaius, 4, 19, introduisit la condictio de omni certa re. L'emploi de la legis actio sacramenti dans notre cas ne soulève même pas, croyonsnous, d'obstacle rationnel pour les auteurs nombreux qui refusent en principe la legis actio aux pérégrins (v. en dernier lieu Schmidt, Zeitschr. d. Sav.-Stift. 9, 139. Wlassak, Processgesetze, 2, 188). En effet, ils l'intenteraient là en vertu d'une loi spéciale expresse, la leur concédant à tous, ratione materiae, en matière de repetundae, comme certains pourrajent même l'avoir reçue plus largement, en vertu de leurs traités, ratione personae; car il nous est impossible d'apercevoir pourquoi la concession expresse de la legis actio serait plus impraticable que celle du commercium : on peut supposer, avec M. Monnnsen, Zeitschr. d. Sav.St. 12, 278, n. 1, une fiction de la cité analogue à celle de Gaius, 4, 37 ; mais cela ne nous parait mème pas nécessaire. La difficulté pourrait encore être supprimée en conjecturant, comme a fait Klenze, fragm. leg. Serviliae, p. 13, que la legis actio sacramenti n'était pas intentée par les pérégrins eux-mêmes, mais par les patroni qu'on les voit avoir avant nos lois 
certain, à notre sens, que, comme on l'admet d'ordinaire, la procédure se soit distinguée, dès le temps des lois Calpurnia et Iunia, des procédures civiles habituelles par la participation du magistrat à la sentence, - pas plus d'ailleurs qu'il n'est directement établi que, comme le veut la doctrine la plus répandue, cette sentence fut rendue par des récupérateurs ${ }^{1}$ ).-En tout cas et quoiqu'il en soit de ces derniers points, le procès est un procès civil intenté en vertu d'une créance civile, devant les tribunaux civils, suivant les formes des procès civils. Mais, cela étant, on ne peut comprendre qu'il s'intente

(Tite-Live, 43, 2) et après elles (lex Acilia ligne 9); seulement on fait alors une exception à la règle Nemo alieno nomine lege agere potest pour en éviter une à l'idée que la legis actio n'appartient qu'aux citoyens.

1) Il n'y a de textes positifs ni sur le premier point ni sur le second. Quant au second, un argument propre, qui nous paraît sérieux sans être absolument décisif, peut être tiré des récupérateurs précédemment nommés pour examiner les réclamations de sujets et d'alliés admises à titre isolé par le sénat ('Tite-Live, 43, 2, par exemple). Par ailleurs on est sans doute surtout déterminé, pour l'un et l'autre, par le texte fondamental de Cicéron, Brut. 27, 106 (p. 42, note 4) qui rattache à la loi Calpurnia l'origine des quaestiones perpetuae. Nons n'objecterons pas, avec A.W.Zumpt, Griminalrecht, 2, 1, p. 6 et ss., que le texte met la loi Calpurnia et les quaestiones dans un rapport de concomitance et non pas de cause ì effet; car on ne voit pas quelle source autre que la loi Calpurnia aurait pu faire la création à cette époque. Mais une quaestio perpetua n'est, par opposition aux quaestiones extraordinaires antérieures, qu'une quaestio permanente. Pour qu'elle ait existé, il a suffi qu'un magistrat, le préteur pérégrin, fut désormais chargé à titre permanent d'accueillir les réclamations qui n'étaient antérieurement renvoyées devant un magistrat que par des décisions isolées. Cela n'empêche pas l'instance d'être, comme les instances civiles, divisée en deux phases: la preuve en est dans le sacramentum. Cela n'empêche pas davantage que le magistrat n'ait pu, tout au moins dans le principe, y avoir, comme dans les autres instances civiles, son rôle réduit à la phase dı ius. Cela n'implique même pas forcément que le procès ait été soumis à des récupérateurs. Sans vouloir rien affirmer sur un régime au sujet duquel nous n'avons pas de témoignages, il nous semblerait concevable que la première loi tout au moins se fut à pen près hornée à donner à tous les pérégrins et les alliés le droit d'agir par sacramentum en matière de repetundae qu'ils n'avaient certainement pas auparavant de droit commun, comme l'avaient les citoyens. Cf. par exemple l'opposition encore faite par Gicéron à propos d'une bi de repetundis postérieure, Div. in Q. Crecilium, 5, 17. 
par voie de sacramentum qu'à condition que la procédure dont le sacramentum est la forme principale, la procédure des Actions de la loi, soit encore à ce moment la seule procédure civile en vigueur, qu'à condition que la procédure par formules entre laquelle et la procédure des Actions de la loi on a eu le choix depuis la loi Aebutia, n'existât pas encore. Si cette procédure plus commode, à laquelle l'accès des pérégrins ne semble pas avoir jamais fait difficulté, avait déjà existé, on eut peut-être exclusivement accordé aux pérégrins visés par nos lois le droit de plaider par formules au lieu de leur ouvrir dans des conditions exceptionelles cette procédure des Actions de la loi qui n'a certainement pas toujours été accessible à la totalité des pérégrins. Tout au moins on ne leur eut pas ouvert la plus étroite et fermé la plus large à une époque où les deux auraient déjà coexisté pour les citoyens.

L'existence du sacramentum en matière de repetundae est la preuve décisive que les premières lois.sur les repetundae trouvèrent encore la procédure des Actions de la loi seule en vigueur. C'est un point qui ne peut guère être contesté, si l'on considère le caractère rigoureusement privé des premiers procès de repetundae. Il l'a cependant été avec une grande assurance, lans des ouvrages qui ne sont pas tous des ouvrages anciens. Mais c'est précisément par suite d'une méconnaissance grave du caractère des poursuites repetundarum et plus largement de l'histoire des quaestiones perpetuae. Il suffit, pour s'en convaincre, de parcourir les réfutations dédaigneuses adressées depuis un peu plus de cinquante ans à la petite phrase par laquelle Heffter a indiqué dès 1827 l'importance des lois Calpurnia et Iunia pour la détermination de la date de la loi Aebutia ${ }^{1}$ ). Il y a des auteurs, comme Bethmannn-Hollweg et M. Jörs, qui se contentent d'affirmer que »la loi Calpurnia n'est pas une raison de mettre la loi Aebutia après 605 « ou que »l'introduction de la procédure des repetundae de la loi Calpurnia par une legis actio sacramenti ne donne pas de terme de discussion précis «. Il y en a de plus explicites qui disent, comme Puchta que »l'identité du sacramento agere $\rho x$

1) Heffter, Gai iurisconsulti institutionum comm. IV, Berlin, 1827, p. 23. 
lege Calpurnia et du sacramento agere ex lege XII tabularum « est une supposition gratuite, ou, comme M. Kuntze qu' »il n'y a pas de conclusion à tirer du droit criminel « ${ }^{1}$ ). Les uns et les autres partent de l'irée, excusable chez les plus anciens, surprenante chez les plus modernes, que les poursuites des lois Calpurnia et Iunia sont des poursuites criminelles comme celles déférées aux comices judiciaires des premiers siècles de la République ou aux tribunaux répressifs du temps de l'Empire. $\mathrm{Si}$, en réalité, on est en présence d'actions civiles en répétition et non pas de procès criminels de concussion, on ne conclut pas du droit criminel au droit civil, mais du droit civil au droit civil; on n'identifie pas gratuitement deux ex sacramento agere sans rapports, on reconnaît, dans sa fonction normale et régulière, un ex sacramento agere unique, qui se présente partout avec la même physionomie et le même caractère, le seul que connaissent les textes juridiques, celui de la legis actio per sacramentum $^{2}$ ); et alors il fournit pour notre discussion un terme que nous considérons non seulement comme précis, mais comme décisif $:$ il fournit une raison excellente de mettre la loi Aebutia après l'an 605 , et même un peu plus tard, après la date incertaine plus récente de la loi Iunia. Car ces lois prouvent qu'à leur époque les créanciers n'avaient pas encore le choix entre la procédure din sacramentum et celle des formules, et ce choix a existé à partir de la loi Aebutia ${ }^{3}$ ).

1) Bethmann-Hollweg, Civilprozess, 2, §55, 2. Puchta, Inst. 1, $\$ 80$, e. Kuntze, Exkurse, p. 248. Jörs, Rechtswiss. 1, 288, 1. Zimmern, 2 , $\S 35,1$, à la réfutation duquel renvoie Rudorff, $1, \S 44,15$, indique aussi l'idée qu'on ne pourrait argumenter de la loi Calpurnia, parce qu'il s'agirait là d'une procédure civile liée à un iudicium publicum, mais il y ajoute d'ailleurs plusieurs confusions. - ${ }^{2}$ ) Le seul auteur qui, à notre connaisance, ait tenté de démontrer l'indépendance du sacramentum des Jois Iunia et Calpurnia par rapport à la legis actio sacramenti, A. W. Zumpt, Criminalrecht, 2, 1, 42 et ss. 1, 2, 286 et ss., n'y arrive qu'en confondant le sacramentum avec les sponsiones de procédure employées pour saisir l'autorité judiciaire d'un différend et qui du reste elles-mêmes n'ont $\mathrm{pu}$, croyons-nous, anciennement fonder d'autre voie de droit que la legis actio sacramenti. - ${ }^{3}$ ) L'argument a la même valeur pour ceux qui, comme M. Schulin, p. 541, admettent que la loi Aebutia a aholi le sacramentum in personam. Au contraire, sans perdre toute portée, il n'aurait pas la même rigueur pour ceux qui considèrent la legis actio sacramenti comme ayant continué après la loi Aebutia 
Nous ne connaissons pas de preuve plus récente de la domination exclusive de la procédure des Actions de la Loi. On pourrait, quoique à notre connaissance on ne l'ait pas encore fait, chercher un argument analogue à celui que nous avons tiré de la loi Calpurnia dans le chapitre de la loi osque de Bantia relatif aux procès civils ${ }^{1}$ ). Là encore il n'est question que de la plus ancienne procédure, soit généralement de la legis actio, soit plus spécialement de la manus iniectio, de la manus iniectio pro iudicato, semble dire le texte, peut-être plutôt de la manus iniectio iudicati. Mais nous croyons impossible de tirer de là un renseignement pratique sur la date de la loi Aebutia. D'abord l'époque du monument est incertaine : tout ce qui nous paraît établi jusqu'à présent, c'est qu'il est nécessairement postérieur à l'an 570 et antérieur à l'an 636, qu'il se place entre la seconde moitié du VI e siècle et la première moitié du $\mathrm{VII}^{\mathrm{e}}{ }^{2}$ ). Ensuite, bien que ce monument, qui semble un statut donné par une autorité romaine à la ville de Bantia, formule en général des règles voisines des règles romaines, rien ne prouve une idlentité absolue de régime, rien ne prouve par exemple que la procédure traditionnelle la

à être le seul moyen d'intenter telle ou telle action personnelle. Mais leur doctrine a été, selon nous, réfutée par M. Wlassak, Processgesetze, 1, 67 et s. Le texte de Cicéron, pro Roscio, 5, 14, opposé par M. Voigt, Berl. phil. Wochenschr. 1888, p. 1414, ne prouve rien. - L'idée selon laquelle le sacramentum des repetundae implique l'inexistence de la loi Aebutia n'a pas été reprise expressément depuis Heffter. Cependant M. Pernice, qui, avons-nous vu, est de ceux qui placent la loi à l'époque la plus récente, a fait remarquer très justement, Zeitschr. d. Sav.-St. 7, 1 , p. 154 , n. 1, l'accomodante légèreté avec laquelle Puchta se débarasse de l'argument de la loi Acilia. M. Mommsen, auquel nous avons communiqué notre sentiment sur l'importance du renseignement fourni par cette loi nous a écrit : "Je pense avec vous que la loi Aebutia est certainement postérieure à l'époque où la l. a. sacramento a servi pour Jes repetundae $\ll$.

1) Lignes 23 - 27. Nous ne reproduisons pas le texte qu'on trouvera dans Bruns, fontes ${ }^{5}$, p. 46 et ss. et dans nos textes de droit romain, p. 26. 27, avec des traductions peu divergentes, $\left.-{ }^{2}\right)$ M. Mommsen, Staatsrecht, $3,702=$ tr. fr. $6,2,334$, place pourtant ce statut local à l'époque des Gracques. Cela supprimerait la première ohjection, mais renforcerait la seconde, qui est d'autant plus solide qu'il s'ayit d'institutions plus récentes à Rome. 
plus archaïque n'ait pas pu subsister seule dans les petits centres italiques quand une procédure nouvelle était déjà expérimentée à côté d'elle à Rome; ce qui nous dispense de chercher jusqu'à quel point le texte de la loi de Bantia qui prouve l'existence des Actions de la loi en prouverait l'existence exclusive.

III.

La loi Aebutia n'existe pas avant l'année 605 de Rome ni même avant la date ignorée plus récente de la loi Iunia. En revanche, elle existe déjà certainement aux environs de l'an 629 et de l'an 631 . Elle se place donc surement entre 605 et 630 , un peu après la première date, un peu avant la seconde, en somme dans le premier tiers du VII e siècle. Et cette détermination que nous avons uniquement tirée de l'examen individuel des témoignages isolés, peut s'appuyer sur un témoignage muet plus général et d'une singulière puissance, sur le rapprochement instructif des textes juridiques antérieurs et postérieurs à la période dans laquelle nous plaçons la loi, des textes postérieurs dans lesquels on voit surgir à la fois de tous côtés les témoins de la procédure nouvelle, des textes antérieurs dans lesquels il est impossible d'en apercevoir une trace.

On pourrait être tenté d'attribuer la différence à la fréquence inégale des premiers et des seconds. Ce serait une erreur. Nous avons, au VI ${ }^{e}$ siècle et au début du VII ${ }^{e}$, des citations des Catons, de P. Mucius Scaevola, consul en 621, de M'. Manilius, cos. en 605 et du préteur M. Iunius, leur contemporain, comme nous en avons un peu plus tard de P. Rutilius Rufus, de M. Livius Drusus et de Q. Mucius Scaevola. Par exemple en laissant de côté Q. Mucius Scaevola, que les jurisconsultes postérieurs ont invoqué avec une fréquence exceptionnelle, nous avons de M'. Manilius une dixaine de citations relatives en partie à la matière de la vente et des stipulations de garantie ${ }^{1}$ ). Il n'y en a pas une qui implique l'existence de la loi Aebutia, d'édits prétoriens, d'actions prétoriennes, ni même de contrats consensuels. Nous en avons sept à huit de P. Rutilius Rufus ${ }^{2}$ ). Sur sept il y en a une

1) M. Lenel, Pal. 1, pp. 589. 590, en relève dix. - ${ }^{2}$ ) M. Lenel, Pal. 1, pp. 185-188, en a accueilli, au texte quatre, auxquelles il faut 
relative à la création d'actions prétoriennes, une autre relative à l'édit sur les operae : deux preuves indéniables de l'existence de la procédure nouvelle. Et ce qui augmente la force du rapprochement, c'est qu'on pourrait aisément l'étendre du domaine étroit de la jurisprudence à d'autres sphères. Ainsi, pour prendre seulement un domaine tout à fait voisin de celui du droit, nous n'avons pas moins de témoignages sur l'histoire de l'éloquence judiciaire dans la dernière moitié du VI ${ }^{*}$ siècle que dans la première moitié du VII ${ }^{e}$. Nous avons par exemple beaucoup plus d'allusions, expresses ou indirectes, aux plaidoyers de Caton le Censeur né en 520, mort en 605, qu'à ceux de L. Licinius Cl'assus, l'orateur, né en 614, mort en 663. On n'a pas encore trouvé un plaidoyer de Caton qui porte trace de la procédure nouvelle. L. Crassus a tout au moins plaidé sur une action emti de bonne foi ${ }^{1}$ ). En considérant même tout l'ensemble de la tradition, nous sommes assurément mieux informés sur l'histoire du Vl ${ }^{\circ}$ siècle, pour laquelle, au moins jusqu'en 587, nous avons Tite-Live, pour une partie de laquelle aussi nous avons Polybe, que nous ne le. sommes sur les temps troublés qui vont ensuite jusqu'à Sulla. Sans doute on peut comprendre à la rigueur que les annales qui nous ont été transmises ne parlent pas de la loi, - en dépit du caractère populaire que lui attribue Gaius et qui est une objection ${ }^{2}$ ), - quand bien même elle se placerait dans

joindre les passages de Gaius, d'Ulpien et de Macrobe, signalés par lui p. 185, note 1. L'argument est encore plus frappant si l'on admet avec M. Mommsen, Symbolae Bethm.-Hollw. obl. p. 92, que les fr. 1-3 ne sont pas de notre Rutilius et que par conséquent nous avons seulement de lui les quatre autres textes.

1) Cicéron, de off. 3, 6, 67:M. Marius Gratidianus, propinquus noster, C. Sergio Oratae vendiderat aedes eas, quas ab eodem ipse paucis ante annis emerat. Eae serviebant, sed hoc in mancipio Marius non dixerat. Adducta res in iudicium est. Oratam Crassus, Gratidianum defendebat Antonius. Ius Crassus urguebat $\gg$ quod vitii venditor non dixisset sciens, id oportere praestari «, aequitatem Antonius, etc. Cf. Bechmann, Kauf, 1, 653. Bekker, Aktion. 1, 164. Un autre plaidoyer de Crassus (Cicéron, Brut. c. 39, 145. c. 53) est prononcé au profit d'un personnage auquel a été accordée la honorum possessio prétorienne, si l'on consent, comme il est raisonnable, à rapporter au même procès le texte de Cicéron, de inv. 2, 21,62. $-{ }^{2}$ ) Tite-Live, 4, 28, parle de la 
la période pour laquelle nous avons les sources les plus complètes. Mais cela s'explique encore bien mieux si elle date du temps pour lequel nous n'avons plus ni Polybe, ni TiteLive, par exemple du temps des Gracques où le motif que lui donne Gaius pourrait s'accorder avec l'initative du premier ${ }^{1}$ ) et pourrait aussi non moins commodément rentrer dans le cercle des réformes partielles accomplies entre le premier et le second.

On a tant abusé, en histoire du droit privé, des considérations faciles tirées des circonstances politiques que nous avons scrupule à insister sur cet ordre d'idées. Il nous faut cependant, afin de ne pas laisser notre démonstration incomplète, noter que la tendance populaire de la réforme s'accorde avec l'esprit démocratique qui souffle de la chute de Carthage à la mort de Gaius Gracchus; que cette substitution de l'écriture aux paroles se concilie fort bien avec le mouvement qui a fait établir le vote écrit dans les comices électoraux en 615 , dans les comices judiciaires en 617 et dans les comices législatifs en 623 et qui fut achevé en 647 par la loi sur la perduellio de L. Coelius Caldus ${ }^{2}$ ); qu'elle présente aussi une certaine symétrie avec l'évolution qui, de l'an 605 à Sulla, remplaça les anciennes poursuites criminelles par la procédure des quaestiones ${ }^{3}$, - trois données qui conduisent au premier tiers ou à la première moitié environ du VII ${ }^{*}$ siècle, et dont les dates que nous avons extraites des textes juridiques ne diffèrent donc que par une précision plus grande.

Enfin, si nous avons naturellement omis, dans toute notre discussion, de recourir à ce procédé d'information dérisoire qui consiste à chercher à travers notre chronologie fragmentaire les Aebutii sauvés de l'oubli par le hasard entre lesquels devrait se disputer la paternité de la loi ${ }^{4}$ ), nous pouvons remarquer, à titre

loi Poetelia Papiria. If ne parle pas de la loi Vallia. Mais rien ne prouve que la loi Vallia soit elle-même antérieure à l'an 588 .

1) Le tribunat de 631 du second est exclu non seulement par la denegatio legis actionis de 628 on 629 (p. 31, note 3), mais par l'action mandati probablement in factum demandée à Sex. Iulius en 631 (p. 32, note 2). - 2) V. Mommsen, Staatsrecht, 3, $404=$ tr. fr. 6, 1, 466. 3) Cette idée a été exprimée par M. Schulin, p. 540. - 4) Tite-Live eite, 41,6, un T. Aebutius Carus, préteur de Sardaigne, de 576, qui a été 
tout à fait accessoire, qu'il ne faudrait pas objecter qu'on ne trouve plus, pour la présenter, parmi les magistrats du VII e

antérieurement triumvir col. ded. en $571(39,55)$ et ensuite décemvir pour le partage de l'ager Ligustinus et Gallicus en $581(42,4)$. Il cite aussi, 44,17, un M. Aebutius Elva, préteur de Sicile en 586, qui apparaît chez lui dix ans auparavant, 41, 1, comme tribunus militum en 576. Pighius ajoute, sans indication de source, dans ses annales : en l'an 520 [Varr. 521] deux tribuns de la plèbe, nommés T. et C. Aebutius Carus, au nom desquels il joint mème l'observation : "si non ab his ab alin tamen Aebutio tribuno plebis lata est hoc quasi tempore lex Aebutia de legis actionibus centumviralium causarum, cuius meminit Gellius «; en l'an 571 [Varr. 572], le tribunat de T. Aebutius Carus qu'il suppose avoir été occupé par lui quatre ans avant sa préture de Sardaigne de 575 [Varr. 576]; en 576. 577 [Varr. 577. 578] deux années de prorogation du gouvernement de Sardaigne du même Aebutius Carus; en 577 [Varr. 578] la questure de $M$. Aebutius Elva, le futur préteur de Sicile; enfin en 622 [Varr. 623] une questure de Sicile occupée par un M. Aebutius Elva, fils du même préteur de Sicile, qu'il mentionne ensuite comme proquesteur les deux années suivantes el comme édile curule en 627 [Varr. 628]. Nous relevons ces indications de Pighius parce qu'elles paraissent l'origine d'une version d'ailleurs singulièrement corrompue qui est encore admise dans plusieurs ouvrages. On lit dans Ortolan, Instituts de Justinien, 1,245 (12 e éd. 1884, p. 207) : » On est réduit à chercher dans l'histoire romaine les années où se trouvent des tribuns du nom d'Aebutius et l'on a à choisir entre les années 520 [les tribuns de Pighius], 577 [la propréture attribuée pour la seconde fois par Pighius en 577 de sa chronologie, 578 de Varron, au préteur de Sardaigne de 575 de Pighius, 576 de Varron?], 583 [?? peut-être le préteur provincial de 586, placé dans la chronologie de Pighius en 585, d'où, par une faute de copie ou d'impression, 583, à moins que l'auteur, pris cette fois d'un scrupule, ne se soit reporté à une édition ancienne de TiteLive où il aura trouvé, 44, 17, la date de $583=169$ av. J. C. pour le tirage au sort, mais où il aurait pu voir qu'il s'agissait d'un préteur, qui même était probablement patricien : voir Mommsen, röm. Forsch. 1, 112, 90]. \& Donc trois années de tribunat, dont l'une n'est dûe qu'à Pighius et dont les deux autres sont des années de préture ou de propréture provinciales, copiées et d'ailleurs fort mal copiées chez. Pighius. C'est en partant de ces données que M. Ortolan, ne doutant pas que la loi n'appartienne à l'une des trois années 520, 583 ou 587, rejette la date de 520, comme trop précoce et déclare que l'on doit »préférer l'une des deux années 577 et 583 * ainsi qu'ont fait à son exemple les auteurs cités dans ce sens p. 11, note 2. - M. Padeletti, Storia del diritt. rom. pp. 251. 252, procède d'une manière moins défectueuse, mais aussi peu sûre en argumentant des deux Aebutii de Tite-Live pour placer la loi à la fin du VIe siècle. 
siècle de membres de la famille qui fournit encore des préteurs à la fin du VI ${ }^{e}$. L'argument ne porterait pas précisément à cause du caractère fragmentaire de nos annales. Mais il y a plus. On peut établir positivement qu'il y avait encore des Aebutii dans la carrière des magistratures à une époque postérieure à celle où nous plaçons la loi. Nous avons une preuve matérielle qu'un Aebutius plébéien a encore occupé le tribunat après le temps des (iracques. C'est la loi Aebutia ou plus exactement le plébiscite Aebutien qui est cité par Cicéron comme ayant léfendu de nommer à une magistrature extraordinaire tant celui qui en avait proposé la création que ses collègues, ses parents et ses alliés ${ }^{1}$ ). Voilà un Aebutius, sans doute un Aebutius Carus, qui a été tribun au VII ${ }^{\circ}$ siècle! Au reste, ce n'est pas l'auteur de notre loi, puisque le plébiscite proposé par lui ne peut guère être placé avant la réaction contre C. Gracchus ${ }^{2}$ ) et que la procédure nouvelle existait alors déjà.

Si l'on voulait à tout prix attacher la loi sur la réforme de la procédure à un nom propre, on pourrait plutôt songer à l'Aebutius qui est indiqué par Pighius, dans sa numération en retard d'un an sur celle de Varron, comme questeur en 622 , proquesteur les deux années suivantes et édile curule en 627. En admettant que ces indications soient exactes et qu'il fut plébéien ${ }^{3}$ ), il aurait pu être tribun du peuple en 625 de Pighius et des fastes, 626 de Varron, et proposer notre loi dans son année de tribunat, entre le 10 décembre 625 et le 9 décembre 626. Mais Pighius le représente comme appartenant à la branche, probablement patricienne, des Aebutii Elvae et son existence elle-même n'est appuyée que par l'autorité du bon annaliste qui a trop inventé pour qu'on le

1) Cicéron, de l. agr. 2, 8, 21 : leges sunt veteres ... tribuniciae ... Licinia ... atque altera Aebutia, quae non modo eum qui tulerit de aliqua curatione ac potestate, sed etiam collegas eius cognatos affines excipit, ne eis ea potestas curatiove mandetur. - ${ }^{2}$ ) Cf. Mommsen, Staatsrecht, 1, 501, n. $2[=$ tr. fr. 2, 151, n. 2.] 2, 630, n. 1. - 3) Si l'on admettait que la répartition de l'édilité curule entre les patriciens aux années impaires de Varron et les plébéiens aux années paires, qui fonctionne au VI e siècle et qui a disparu en 665 (Mommsen, röm. Forsch. 1, 97 et ss. Staatsrecht, 2, 402), existait encore à cette époque, on pourrait invoquer dans ce sens l'édilité curule occupée par lui en l'an 627 des fastes, 628 de Varron. 
croie sur parole. D'ailleurs le tribunat d'un Aebutius plébéien fut-il avéré pour l'an 626 , nous le relèverions uniquement comme une coüncidence. Ce n'est pas du hasard des noms propres, c'est des données juridiques, que nous avons essayé de rassembler dans ce travail, qu'on peut, croyons-nous, exclusivement et sûrement tirer la date de la loi.

\title{
III. \\ Der Process des Quinctius \\ und \\ C. Aquilius Gallus.
}

\author{
Von \\ Herrn Dr. Bernhard Kübler \\ in Berlin.
}

Für das juristische Verständniss der Rede, welche Cicero für den P. Quinctius gehalten hat, hat Niemand mehr geleistet, als Keller in seinen methodisch vollendeten Semestria ad M. Tullium Ciceronem. Nicht als ob er in allen Dingen das Richtige getroffen hätte; aber die Grundlage, auf der alle Späteren fussen, ist doch von ihm geschaffen und viele Punkte sind von ihm zum ersten Male richtig erkannt und dargestellt worden. Wenn man dann auf diesem Fundament rüstig weiter gebaut und sich dabei nicht gescheut hat, auch manchen der von Keller errichteten Pfeiler wieder einzureissen, so hat man nur seinen eigensten Wunsch erfüllt, da es gerade seine $A b-$ sicht war, durch seine Arbeit Andere zur Prüfung, Berichtigung und Fortsetzung des von ihm Begonnenen zu veranlassen. Die Juristen, an die seine Aufforderung in erster Linie gerichtet war, haben den Process mehrmals, theils für sich, theils in anderm Zusammenhang behandelt ${ }^{1}$ ); die Philologen aber haben

1) Recensionen des Keller'schen Werkes von Mommsen in der Zeitschr. f. Alterthumswissenschaft 1845 p. $1084 \mathrm{ff}$. und Bachofen in Richters Krit. Jahrb. Bd. 12 (1842) p. 961 ff. Ausserdem: Frey, der Rechtsstreit zwischen P. Quinctius und S. Naevius, Zürich 1852. Derselbe im 